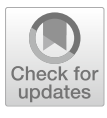

Cite as

Nano-Micro Lett.

(2022) 14:52

Received: 12 October 2021

Accepted: 7 December 2021

Published online: 29 January 2022

(C) The Author(s) 2022

\section{Self-Healing, Self-Adhesive and Stable Organohydrogel-Based Stretchable Oxygen Sensor with High Performance at Room Temperature}

\author{
Yuning Liang ${ }^{1}$, Zixuan $\mathrm{Wu}^{1}$, Yaoming $\mathrm{Wei}^{1}$, Qiongling Ding ${ }^{1}$, Meital Zilberman ${ }^{2}$, \\ Kai $\mathrm{Tao}^{3}, \mathrm{Xi} \mathrm{Xie}^{1}$, Jin $\mathrm{Wu}^{1}$ ه
}

\title{
HIGHLIGHTS
}

- The organohydrogel-based $\mathrm{O}_{2}$ sensor features full concentration detection range (0-100\%), ultralow limit of detection (5.7 ppm), high sensitivity $(0.2 \% / \mathrm{ppm})$, excellent selectivity, tunable response/recovery speeds, good linearity, and room-temperature operation.

- The oxygen sensor can work normally under various extreme environmental conditions, such as low (below -18 ${ }^{\circ} \mathrm{C}$ ) and high (above $\left.40{ }^{\circ} \mathrm{C}\right)$ temperatures, dry $(11.3 \% \mathrm{RH})$, and humid $(90.5 \% \mathrm{RH})$ environments.

- An electrochemical reaction-based mechanism is proposed to elucidate the oxygen sensing behavior of ion-conducting organohydrogel.

\begin{abstract}
With the advent of the 5G era and the rise of the Internet of Things, various sensors have received unprecedented attention, especially wearable and stretchable sensors in the healthcare field. Here, a stretchable, self-healable, self-adhesive, and roomtemperature oxygen sensor with excellent repeatability, a full concentration detection range (0-100\%), low theoretical limit of detection (5.7 ppm), high sensitivity $(0.2 \% / \mathrm{ppm})$, good linearity, excellent temperature, and humidity tolerances is fabricated by using polyacrylamide-chitosan (PAM-CS) double network
\end{abstract}

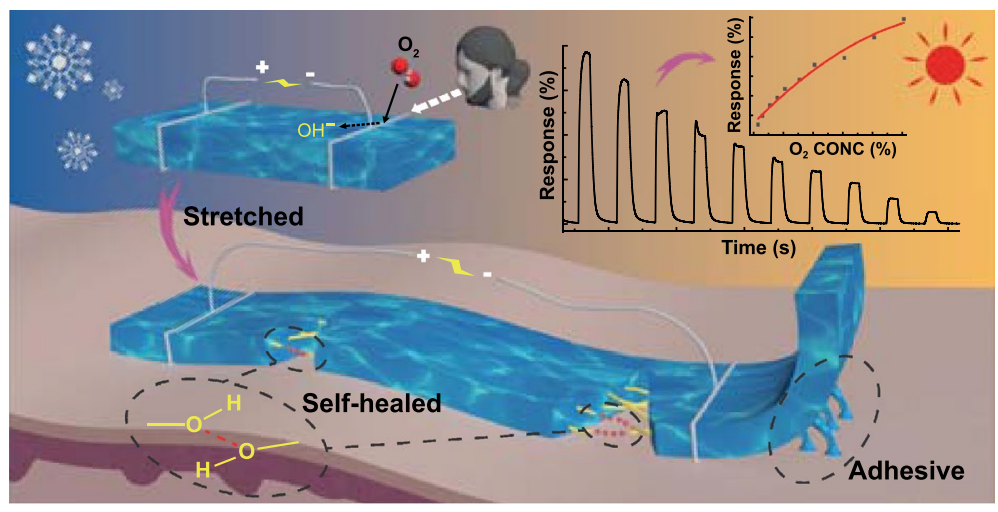
(DN) organohydrogel as a novel transducing material. The PAM-CS DN organohydrogel is transformed from the PAM-CS composite hydrogel using a facile soaking and solvent replacement strategy. Compared with the pristine hydrogel, the DN organohydrogel displays greatly enhanced mechanical strength, moisture retention, freezing resistance, and sensitivity to oxygen. Notably, applying the tensile strain improves both the sensitivity and response speed of the organohydrogel-based oxygen sensor. Furthermore, the response to the same concentration of oxygen before and after self-healing is basically the same. Importantly, we propose an electrochemical reaction mechanism to explain the positive current shift of the oxygen sensor and corroborate this sensing mechanism through rationally designed experiments. The organohydrogel oxygen sensor is used to monitor human respiration in real-time, verifying the feasibility of its practical

$\checkmark$ Jin Wu,wujin8@mail.sysu.edu.cn

1 State Key Laboratory of Optoelectronic Materials and Technologies and the Guangdong Province Key Laboratory of Display Material and Technology, School of Electronics and Information Technology, Sun Yat-Sen University, Guangzhou 510275, People's Republic of China

2 Department of Biomedical Engineering, Faculty of Engineering, Tel Aviv University, 69978 Tel Aviv, Israel

3 Ministry of Education Key Laboratory of Micro and Nano Systems for Aerospace, Northwestern Polytechnical University, Xi' an 710072, People's Republic of China 
application. This work provides ideas for fabricating more stretchable, self-healable, self-adhesive, and high-performance gas sensors using ion-conducting organohydrogels.

KEYWORDS Stretchable oxygen sensors; Organohydrogel; Self-healing; Self-adhesive; Electrochemical reaction

\section{Introduction}

In recent years, with the rapid development of the Internet of Things (IoT) and the growing requirements of people's life quality, various sensors used for monitoring health and environmental quality have gradually entered people's daily lives to enhance people's living standards [1-5]. For instance, gas sensors can be exploited to monitor the content of various gases in the environment [6-9]. Oxygen is the most indispensable gas in human life activities. It participates in the aerobic decomposition of carbohydrates that provide energy for life activities [10]. A low-oxygen environment will cause many adverse physiological reactions to the human body. Specifically, when the oxygen concentration is lower than $17 \%$, it will cause symptoms such as dyspnea, fatigue, and decreased attention. Furthermore, suffocation fatal accidents may even occur when the oxygen concentration is lower than $12 \%[11,12]$. Otherwise, people need oxygen with a concentration higher than that in the air in some specific occasions, such as anesthesia machines and hyperbaric oxygen chambers in hospitals, and oxygen content analysis for aerospace and diving operations [13-15]. Therefore, the monitoring of oxygen with a wide range of concentrations is essential $[16,17]$. In particular, if the oxygen sensor is stretchable, portable, self-healing, and self-adhesive, it can be directly attached to people's clothing and even skin for persistent monitoring and can resist general mechanical damage. This will increase the convenience and prolong the service life of the sensor, which can greatly meet people's daily use requirements for wearable applications [18-21].

However, the gas sensing materials of conventional stretchable gas sensors cannot be stretched, self-healed, and self-adhesive. Instead, these stretchable gas sensors are fabricated by integrating non-flexible gas sensing materials, such as $\mathrm{MoS}_{2}, \mathrm{~V}_{2} \mathrm{O}_{5}, \mathrm{TiO}_{2}$, and graphene, on flexible substrates, such as polydimethylsiloxane (PDMS), ecoflex, and Polyimide (PI) [22-27]. This will complicate the fabrication process. Furthermore, the stretchability of these sensors will also be limited by the substrates [28]. Most importantly, the detection objects of currently emerging stretchable gas sensors focus on toxic gases, such as $\mathrm{NO}_{2}, \mathrm{SO}_{2}$, and $\mathrm{NH}_{3}$, leaving stretchable oxygen sensors undeveloped [16, 29-31]. Among traditional oxygen sensors, people have the most in-depth research on chemiresistor-type sensors based on metal oxide semiconductors (MOS), which are easy to manufacture and therefore cost-effective [32, 33]. However, except for the lack of stretchability and self-healability, these sensors also have the problem of high operating temperature $\left(100-500{ }^{\circ} \mathrm{C}\right)$. Furthermore, it is also difficult for them to achieve a wide detection range, good linearity, and fast response/recovery speeds [34-36]. For example, Xiong et al. prepared an oxygen sensor based on LaOCl-doped $\mathrm{SnO}_{2}$, which has the advantages of working at room temperature (RT) and high selectivity against $\mathrm{H}_{2}, \mathrm{CH}_{4}, \mathrm{NH}_{3}$, and $\mathrm{CO}_{2}$. But its detection range is limited within $100-5000 \mathrm{ppm}$, and the response and recovery speeds are slow. Specifically, the response and recovery time to $250 \mathrm{ppm} \mathrm{O}_{2}$ are 182 and $1315 \mathrm{~s}$, respectively [37]. Although Xu et al. proposed the utilization of two-dimensional $\mathrm{Bi}_{2} \mathrm{O}_{2} \mathrm{Se}$ to detect $\mathrm{O}_{2}$ with an extremely low concentration of $0.25 \mathrm{ppm}$, the sensor failed to display the flexibility/stretchability, linearity, and fast response speed [38]. In addition, some oxygen sensors based on new sensing materials also have limitations [39, 40]. For example, Yeon Hoo Kim and co-workers proposed the utilization of $\mathrm{MoS}_{2}$ nanoparticles to prepare an oxygen sensor, which presented high and linear responses to $\mathrm{O}_{2}$ with a broad range of concentrations. But the sensor needs to be operated at $300{ }^{\circ} \mathrm{C}[12]$. Until now, stretchable, self-healing, self-adhesive, and high-performance (linear, a wide detection range, selectivity, etc.) oxygen sensor working at RT has not yet been reported.

Hydrogel is a polymer material with a three-dimensional polymer network structure formed by chemical or physical cross-linking and swelled in a large amount of water. Many hydrogels have excellent stretchability, ionic conductivity, self-healability, biocompatibility, etc., and have been widely applied to fabricate wearable sensors for the monitoring of various physical and chemical quantities, such as pressure, temperature, strain, humidity, and the like [41-44]. Herein, for the first time, we employ polyacrylamide-chitosan 
(PAM-CS) composite organohydrogel to fabricate stretchable, self-healable, self-adhesive, and high-performance electronic oxygen sensors that can work at RT $\left(27^{\circ} \mathrm{C}\right)$ (Fig. 1a). Compared with traditional oxygen sensing materials, the organohydrogel features high stretchability (up to $1400 \%$ strain), self-healability, and RT operation. Furthermore, its good self-adhesive performance enables it to be directly adhered to clothing or even people's skin for operation without entailing additional adhesive tape, which greatly simplifies the actual fabrication process of the wearable sensor. In addition, a facile soaking and solvent replacement strategy were devised to convert the hydrogel to corresponding double network (DN) organohydrogel with enhanced mechanical strength and moisture retention ability. 1,2-Propanediol (1,2-PD) was chosen for the solvent replacement instead of common glycerol owing to its smaller molecule and better permeability [45]. Thus, it should provide a more efficient solvent replacement process. More importantly, (a)

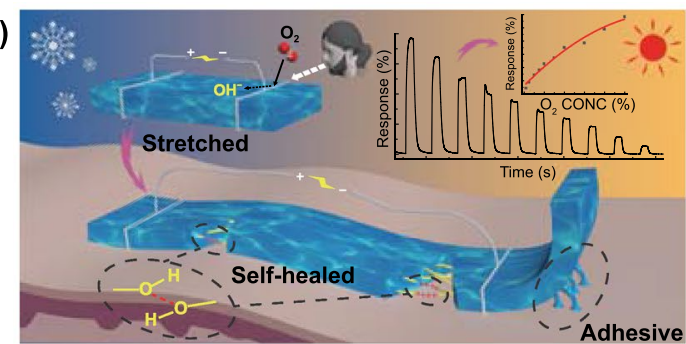

(b)

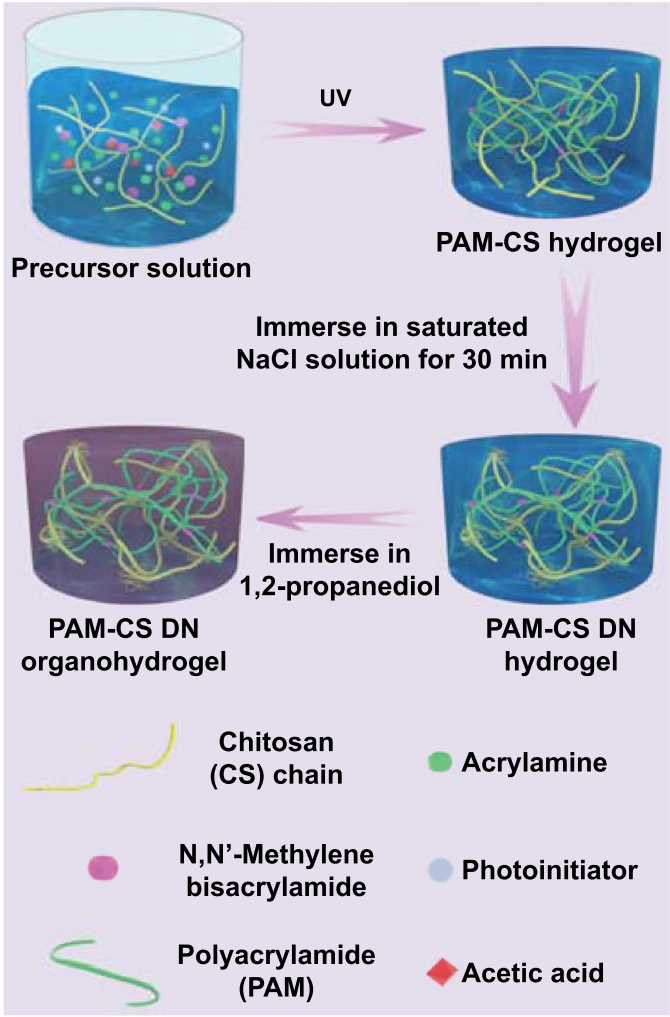

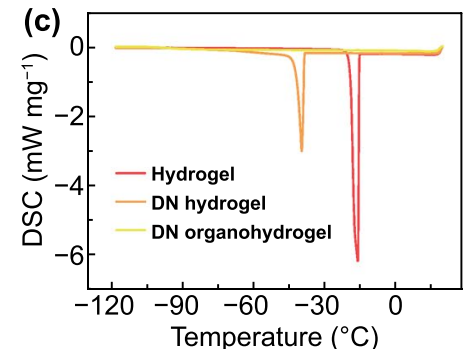
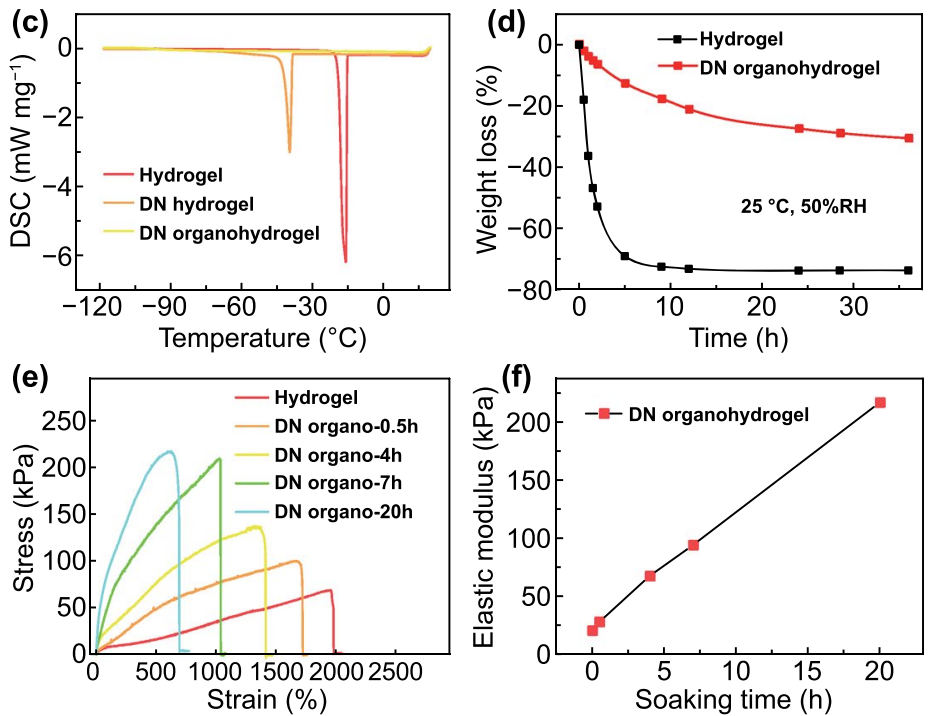

(g)

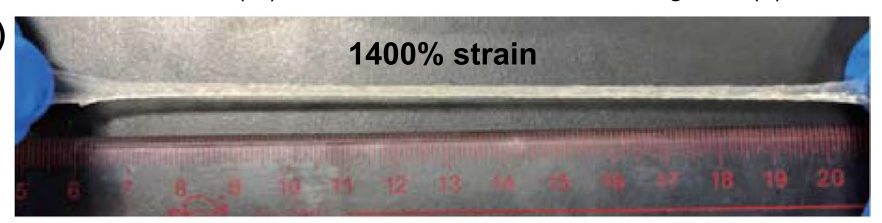

(h)

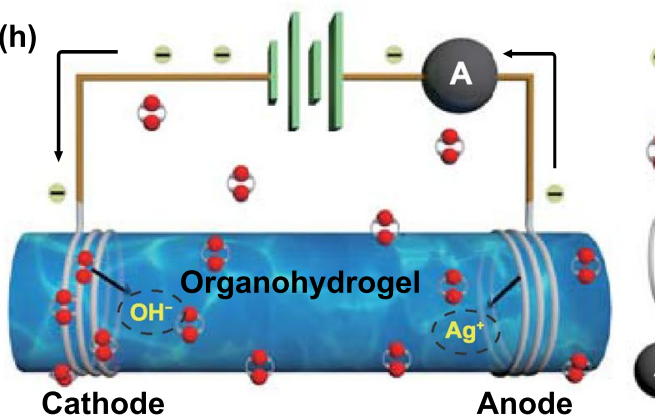

Electronic

Oxygen

Silver wire

Amperemeter

Fig. 1 a Schematic illustrating the organohydrogel-based stretchable, self-adhesive, self-healable oxygen sensors working at room temperature. b Scheme showing the synthesis of PAM-CS DN organohydrogel. c DSC curves of hydrogel, DN hydrogel, and DN organohydrogel. d Time evolution of the weight loss percentage of hydrogel and DN organohydrogel when stored at $25{ }^{\circ} \mathrm{C}$ and $50 \%$ RH. e Stress-strain curves of hydrogel and DN organohydrogels obtained by soaking in 1,2-PD for $0.5,4,7$, and $20 \mathrm{~h}$, respectively. $\mathbf{f}$ Plot of the elastic modulus of DN organohydrogel vs soaking time. g Photograph showing the DN organohydrogel at $1400 \%$ tensile strain. h Schematic illustrating the working principle of the oxygen sensor 
the effect of 1,2-PD on the moisture retention and freezing resistance of hydrogel has rarely been studied. The DN organohydrogel-based oxygen sensor also features full concentration detection range, low limit of detection (LOD), good linearity (within 0-20\% $\mathrm{O}_{2}$ ), high sensitivity, and selectivity. Besides, the sensor can also operate properly in the stretched state and after self-healing, which is not available for previously reported oxygen sensors.

\section{Experimental Section}

\subsection{Synthesis of Hydrogel and DN Organohydrogel}

All chemicals including acrylamide (AM), N,N'-Methylene bisacrylamide (MBA), Irgacure2959 (photoinitiator), chitosan (degree of deacetylation $>90 \%, \mathrm{MW}=30,000$ ), acetic acid, and 1,2-propanediol (1,2-PD) were purchased from Sigma-Aldrich. The PAM-CS composite hydrogel was prepared via a one-pot sol-gel process. Firstly, chitosan $(0.8 \mathrm{~g})$ and acetic acid $(115 \mu \mathrm{L})$ were added to deionized water $(20 \mathrm{~g})$ and stirred magnetically at a speed of $900 \mathrm{rpm}$ for $3 \mathrm{~h}$, so that the chitosan was fully dissolved in water to form a chitosan solution. Then, AM (3.6 g), MBA (0.002 g), and photoinitiator $(0.115 \mathrm{~g})$ were added to the chitosan solution and stirred magnetically for $2 \mathrm{~h}$ to obtain a precursor solution, which formed PAM-CS composite hydrogel after being radiated with ultraviolet (UV) light for $1 \mathrm{~h}$. The hydrogel was soaked in a saturated $\mathrm{NaCl}$ solution for $30 \mathrm{~min}$ to make the chitosan chains form a chain entanglement structure, generating the PAM-CS DN hydrogel. Finally, the prepared DN hydrogel was soaked in 1,2-PD for $10 \mathrm{~h}$ for the solvent replacement, leading to the formation of PAM-CS DN organohydrogel (Fig. 1b).

\subsection{Material Characterization}

The differential scanning calorimetry (DSC) spectra were obtained on a Netzsch DSC-204. The Fourier transform infrared (FTIR) spectra were acquired on a Thermo Scientific Nicolet 6700 FTIR Spectrometer. Field emission scanning electron microscopy (SEM) (ZEISS SUPRA 60) was deployed to analyze the surface morphology and elements of the electrodes. The Instron machine (S6566) was employed to acquire the strain-stress curves and the peel force vs displacement curves.

\subsection{Gas Sensing Characterization}

A homemade gas sensing characterization system was deployed for the gas sensing test at $27{ }^{\circ} \mathrm{C}$ and $74 \%$ relative humidity (RH) if without special indication. An airtight bottle with an air inlet and outlet was utilized as the gas chamber. The sensor was placed in the test chamber, and the $\mathrm{Ag}$ wires were deployed as the electrodes of the sensor and led out of the gas chamber. The Keithley 2400 source meter was exploited to monitor the current in the circuit after a constant DC bias voltage of $0.5 \mathrm{~V}$ was applied. The DIG-III gas distribution system was utilized to control the flow rate of two gas streams, one of which was the mixture of $\mathrm{O}_{2}$ and $\mathrm{N}_{2}$, and the other was pure $\mathrm{N}_{2}$. The two gas streams were mixed with different ratios to obtain specific concentrations of $\mathrm{O}_{2}$. During the test, pure $\mathrm{N}_{2}$ was employed to purge the test chamber before and after feeding $\mathrm{O}_{2}$. To measure the gas sensing properties of sensor at different strains, two binders were utilized to fix the stretched organohydrogel on a glass slide after applying certain tensile strains such as $25 \%, 50 \%$, and $100 \%$.

\section{Results and Discussion}

\subsection{Characterization of Organohydrogel}

The highly stretchable PAM-CS hydrogel was synthesized via a facile one-pot sol-gel process. The details are described in the experimental part. Specifically, the hydrogel was soaked in saturated sodium chloride $(\mathrm{NaCl})$ solution to enable the dispersed chitosan chains to form a chain entanglement structure in the first step, thereby converting the PAM-CS composite hydrogel into a DN hydrogel. Then the DN hydrogel was soaked in 1,2-PD to replace part of the water in the solvent with 1,2-PD, leading to the formation of PAM-CS DN organohydrogel with a water-1,2-PD binary solvent. By comparing the FTIR spectra of hydrogel and DN organohydrogel, it can be seen that the intensities of the $\mathrm{O}-\mathrm{H}$ stretching peak at $3347 \mathrm{~cm}^{-1}$ and $\mathrm{O}-\mathrm{H}$ bending peak at $1040 \mathrm{~cm}^{-1}$ of the DN organohydrogel are higher than that of corresponding hydrogel (Fig. S1). This is attributed to the introduction of 1,2-PD molecules in the hydrogel through the solvent replacement strategy, which is also confirmed by the DSC measurement (Fig. 1c). The DSC spectra show that, compared with the freezing point of untreated 
hydrogel $\left(-16^{\circ} \mathrm{C}\right)$, the DN hydrogel obtained by the soaking strategy displays a much lower freezing point $\left(-40^{\circ} \mathrm{C}\right)$. The freezing point of the untreated hydrogel is lower than $0{ }^{\circ} \mathrm{C}$. This is because the functional groups such as amino and hydroxyl groups on the polymer chain can form hydrogen bonds with water molecules, which increases the content of bound water and thus lowers the freezing point [46, 47]. The decrease in the freezing point of DN hydrogel is attributed to the formation of a chitosan chain entanglement structure with the aid of $\mathrm{NaCl}$, enhancing the stability of the structure. Furthermore, the incorporation of $\mathrm{NaCl}$ also decreases the freezing point due to the ionic hydration effect. On this basis, the anti-freezing performance of the DN organohydrogel has been further boosted by using the solvent replacement strategy to incorporate 1,2-PD in the solvent, as the freezing point has dropped to below $-120^{\circ} \mathrm{C}$. It is because each 1,2-PD molecule contains two hydroxyl groups, which can form strong hydrogen bonds with water molecules, which inhibits the formation of ice crystals at subzero temperatures [48].

Hydrogels are generally prone to lose water, which greatly affects the stability of both hydrogels and related devices. In particular, most gas sensors need to be directly exposed to air, making it difficult for hydrogel-based gas sensors to be practically applied, especially for long-term stable applications [49]. Fortunately, the drying resistance of hydrogel can be greatly enhanced by creating DN organohydrogel, in addition to the anti-freezing capacity [50]. Specifically, when the hydrogel was exposed to a dry environment of $24{ }^{\circ} \mathrm{C}$ and $50 \% \mathrm{RH}$ for $5 \mathrm{~h}$, its mass loss reached as high as $70 \%$. By comparison, the mass loss of DN organohydrogel stabilized at about $30 \%$ within $36 \mathrm{~h}$ under the same circumstance, implying the boosted water retention ability of organohydrogel (Fig. 1d). Comparing the states of hydrogel and DN organohydrogel before and after the drying experiment, it can be found that after $36 \mathrm{~h}$, the hydrogel has completely turned into a hard block and cannot bear deformations such as stretching, while the DN organohydrogel can still withstand $250 \%$ tensile strain (Fig. S2). It verifies that the introduction of 1,2-PD in the solvent can significantly improve the moisture holding capability of the hydrogel. This is because 1,2-PD introduces a large number of hydroxyl groups, which increase the content of bound water by forming strong hydrogen bonds with water molecules [51].
Moreover, compared with the untreated hydrogel, the mechanical strength of DN organohydrogel soaked in $\mathrm{NaCl}$ solution and 1,2-PD can be remarkably promoted at the expense of certain stretchability (Fig. 1e). Furthermore, the longer the hydrogel was soaked in 1,2-PD, the greater its elastic modulus (Fig. 1f). Specifically, the elastic modulus of organohydrogel increased from 20.3 to $208.2 \mathrm{kPa}$ with prolonged immersion time from 0 to $20 \mathrm{~h}$, showing 10.26 times enhanced mechanical strength. This is because the hydroxyl groups of 1,2-PD molecules formed a large number of hydrogen bonds with the hydroxyl or amino groups of the chitosan chains, which greatly strengthened its originally loose chain entanglement structure. Considering the balance between the stretchability and mechanical strength, the best formulation can be obtained by using the soaking time of hydrogel in $\mathrm{NaCl}$ solution and 1,2PD of 0.5 and $7 \mathrm{~h}$, respectively. With this approach, the obtained DN organohydrogel displays the elastic modulus of $94 \mathrm{kPa}$, and meanwhile can withstand more than $1400 \%$ tensile strain (Fig. 1g). In addition to the high toughness, both the hydrogel and DN organohydrogel can withstand severe deformations, such as $180^{\circ}$ bending and $720^{\circ}$ twisting deformations (Fig. S3).

\subsection{Gas sensing Mechanism and Performance}

A two-electrode chemiresistor-type structure was employed to construct the oxygen sensor, in which the organohydrogel and the silver wires at both ends functioned as the solidstate electrolyte and electrodes, respectively, as shown in Fig. $1 \mathrm{~h}$. The gas sensing performance of oxygen sensors was characterized by monitoring the change of current flowing through the organohydrogel under a constant direct-current (DC) voltage of $0.5 \mathrm{~V}$ when exposed to pure nitrogen $\left(\mathrm{N}_{2}\right)$ and specific concentrations of oxygen alternately. We propose the redox reactions occurring at the hydrogel-electrode interface to explain the gas responsive behaviors of the organohydrogel sensor. The entire system can be regarded as an electrolytic cell. When the organohydrogel is in a pure $\mathrm{N}_{2}$ environment, the steady-state current flowing through it is $\mathrm{I}_{0}$. When the sensor is exposed to a test gas containing a specific concentration of oxygen, the oxygen gains electrons at the cathode, and a reduction reaction occurs, leading to the generation of $\mathrm{OH}^{-}$: 
$\mathrm{O}_{2}+2 \mathrm{H}_{2} \mathrm{O}+4 e^{-} \rightarrow 4 \mathrm{OH}^{-}$

Meanwhile, the silver electrode loses electrons at the anode, and an oxidation reaction occurs to generate $\mathrm{Ag}^{+}$:

$\mathrm{Ag}-e^{-} \rightarrow \mathrm{Ag}^{+}$

$\mathrm{Ag}^{+}+\mathrm{Cl}^{-} \rightarrow \mathrm{AgCl}$

The response here is defined as $\left(I_{O_{2}}-I_{0}\right) / I_{0}$, where $I_{O_{2}}$ and $I_{0}$ are the stabilized currents when the sensor is exposed to $\mathrm{O}_{2}$ and pure $\mathrm{N}_{2}$, respectively. It is found that the contact surface area has little effect on the magnitude of response of the sensor. This is because the larger contact surface area enabled by increasing the coiling number of the $\mathrm{Ag}$ wire promotes both the electrochemical reactions and the initial current (baseline of the response curve), leading to the increase in both $I_{O_{2}}$ and $I_{0}$ (Fig. S4). The two effects offset each other, leading to a similar response. Figure 2a shows the dynamic response curve of the sensor upon exposure to pure $\mathrm{N}_{2}$ and $1 \% \mathrm{O}_{2}$ repeatedly and alternately. It can be seen that the sensor produced a constant and high response of $3200-1 \% \mathrm{O}_{2}$, indicating its excellent repeatability (Fig. 2b). The hysteresis curves of the sensor are shown in Fig. S5. When the oxygen concentration increases from 1 to $16 \%$ and then decreases to $1 \%$ at the same rate, the calibration curves in the ascending and descending $\mathrm{O}_{2}$ concentrations are highly similar. As such, the sensor displays a low hysteresis of $0.4 \%$, which also proves its good reproducibility. The response time is defined as the time taken for $90 \%$ of the total current variation after a specific concentration of $\mathrm{O}_{2}$ is continuously introduced; while the recovery time is denoted as the time required to recover to $10 \%$ of the original current after removing $\mathrm{O}_{2}$ in the chamber using $\mathrm{N}_{2}$ purge [52]. As such, the response and recovery time in the detection of $1 \% \mathrm{O}_{2}$ are 39.9 and 63.7 s, respectively (Fig. 2c). Compared with previously reported oxygen sensors based on other sensing materials, such as multi-walled carbon nanotubes (MWC$\mathrm{NTs}$, $\mathrm{ZnO}, \mathrm{TiO}_{2}$, and $\mathrm{SnO}_{2}$, the response and recovery time of the PAM-CS DN organohydrogel $\mathrm{O}_{2}$ sensors are equivalent or shorter (Table S1).

Figure S6a shows the dynamic curve of current versus time when the sensor was exposed to $2 \%-100 \% \mathrm{O}_{2}$, and the corresponding dynamic response curve of the sensor is shown in Fig. 2d. It can be observed that when the sensor was exposed to $\mathrm{O}_{2}$, the current through it increased immediately. Furthermore, the higher the $\mathrm{O}_{2}$ concentration, the greater the current through the sensor, and thus the greater response obtained. It demonstrates the capability of the organohydrogel sensor to discriminate different $\mathrm{O}_{2}$ concentrations within a wide range. This is because $\mathrm{O}_{2}$ participated in an electrochemical reaction at the electrode of the sensor, which generated a Faraday current. The higher the oxygen concentration, the more $\mathrm{O}_{2}$ was involved in the electrochemical reaction, and the greater the Faraday current generated, leading to a higher response. Since the test time is as long as $6 \mathrm{~h}$, the weight loss of the organohydrogel itself may also decrease the performance of the oxygen sensor. To eliminate this interference and get a rigorous conclusion, a durability test was also conducted, which was to expose the sensor to pure $\mathrm{N}_{2}$ and $1 \% \mathrm{O}_{2}$ repeatedly and alternately for as long as $6 \mathrm{~h}$ (Fig. S6b). It can be seen that the response of the sensor to $1 \% \mathrm{O}_{2}$ is relatively stable within $6 \mathrm{~h}$ with an error of less than $4 \%$, which proves the durability of the organohydrogel oxygen sensor during long-term testing. In addition, the sensor can maintain a relatively stable current when exposed to the air for more than $8 \mathrm{~h}$, which further verifies its good stability (Fig. S6c).

In addition to high $\mathrm{O}_{2}$ concentrations, the sensor also showed appreciable responses to low concentrations of $\mathrm{O}_{2}$ (40-1600 ppm), as shown in Fig. 2e. Moreover, there is a strong positive correlation between the response and oxygen concentration within 2-100\% $\mathrm{O}_{2}$ (Fig. 2f). Notably, there is a significant linear relationship between the response and $\mathrm{O}_{2}$ concentration within 2-20\% (the green box in the inset of Fig. 2f). Figure $2 \mathrm{~g}$ further depicts that the sensor maintained good linearity at low $\mathrm{O}_{2}$ concentrations $(40-1600 \mathrm{ppm})$. Note that $40 \mathrm{ppm} \mathrm{O}_{2}$ is the low-limit $\mathrm{O}_{2}$ concentration provided by our current experimental setup, but can be well perceived by this sensor. The good linearity within a wide range of $\mathrm{O}_{2}$ concentrations is advantageous for practical application. The slope of response versus concentration curve within $0-20 \% \mathrm{O}_{2}$ was calculated as $0.2 \% / \mathrm{ppm}$, which was the sensitivity. The low theoretical LOD of $5.7 \mathrm{ppm} \mathrm{O}_{2}$ was further obtained by calculating the root mean square deviation of the noise from the dynamic response curve and the sensitivity (Fig. S7a-b, Table S2) [53]. In general, the oxygen sensor features excellent repeatability, a full concentration detection range, linearity within $0-20 \% \mathrm{O}_{2}$, high sensitivity $(0.2 \% / \mathrm{ppm})$, and low LOD (5.7 ppm), and 

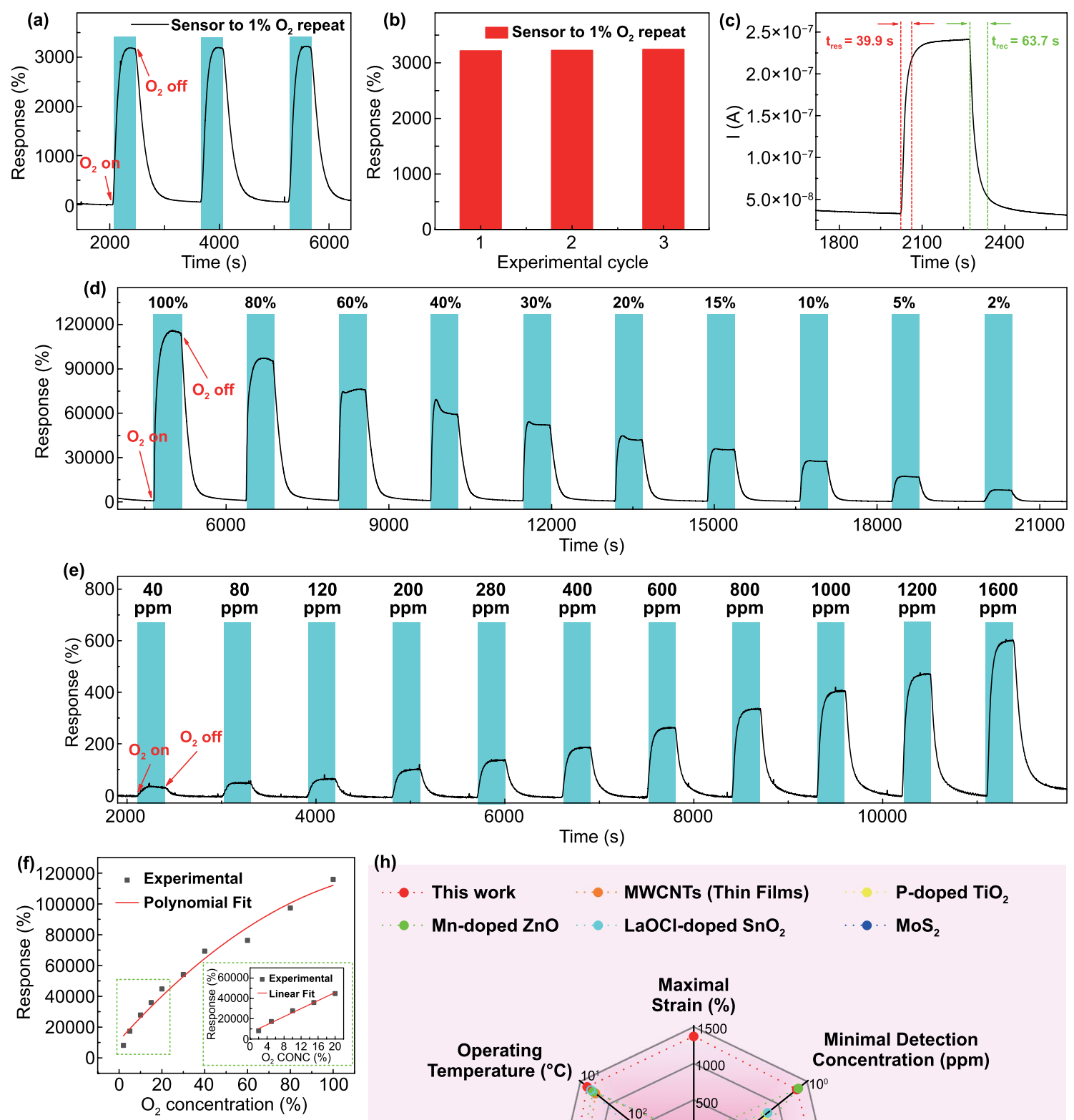

(h)
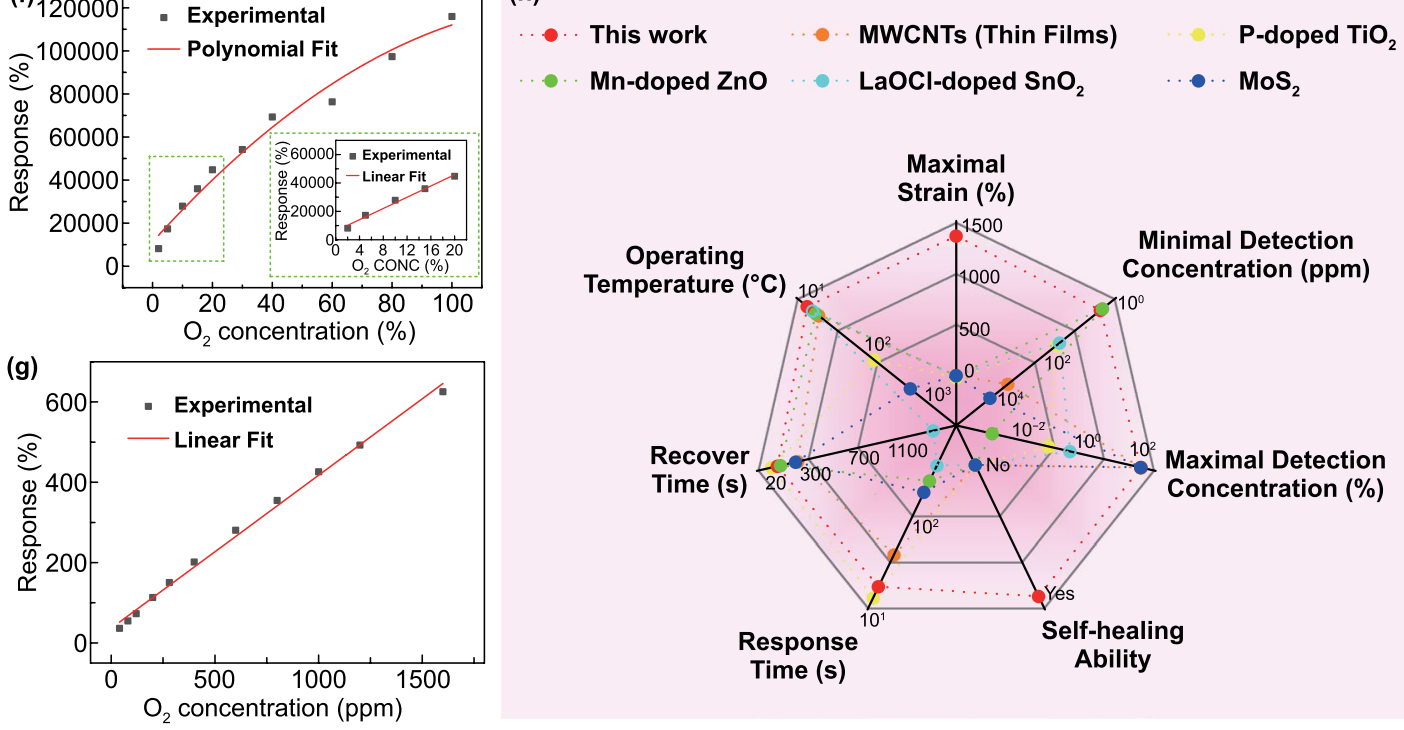

Fig. $2 \mathrm{O}_{2}$ sensing properties of the organohydrogel sensor. a Time-dependent response of the sensor to $1 \% \mathrm{O}_{2}$ in three experimental cycles. The blue and white areas represent the "on" and "off" states of test gas in the gas chamber. b Quantitative response extracted from a versus experimental cycle. c Investigation of the response and recovery time in the detection of $1 \% \mathrm{O}_{2}$. d, e Time-dependent responses of the sensor upon exposure to $\mathrm{O}_{2}$ with wide ranges of high (2-100\%) and low (40-1600 ppm) concentrations, respectively. $\mathbf{f}$ The polynomial fit of responses versus $\mathrm{O}_{2}$ concentration within 2\%-100\%. The green box in the inset shows a linear fit of response versus $\mathrm{O}_{2}$ concentration from 2 to $20 \%$. $\mathrm{g}$ The linear fit of responses versus $\mathrm{O}_{2}$ concentration within $40-1600 \mathrm{ppm}$. h The radar chart comparing the performance of this $\mathrm{O}_{2}$ sensor with that of other $\mathrm{O}_{2}$ sensors 
therefore compares advantageously with existing oxygen sensors (Fig. 2h, Table S1).

\subsection{Self-Healing and Self-Adhesive Properties}

In actual wearable applications, the sensor may be suffered from external forces and break. Fortunately, the self-healing ability of the organohydrogel can perfectly solve this problem, which is a unique advantage of the organohydrogel-based sensor $[48,54]$. To investigate the electrical self-healability of organohydrogel, the organohydrogel, a blue LED indicator, and a 3 V DC voltage source were connected in series to form a circuit (Fig. 3a). The LED indicator was lighted before cutting off the conductive organohydrogel, while went out after being completely severed. Notably, the LED indicator was lightened again after reconnection of the cut organohydrogel at RT, suggesting the ready self-healability of the electrical conductance. To further quantitatively characterize the self-healing efficiency of the organohydrogel, the Keithley 2400 was used to measure
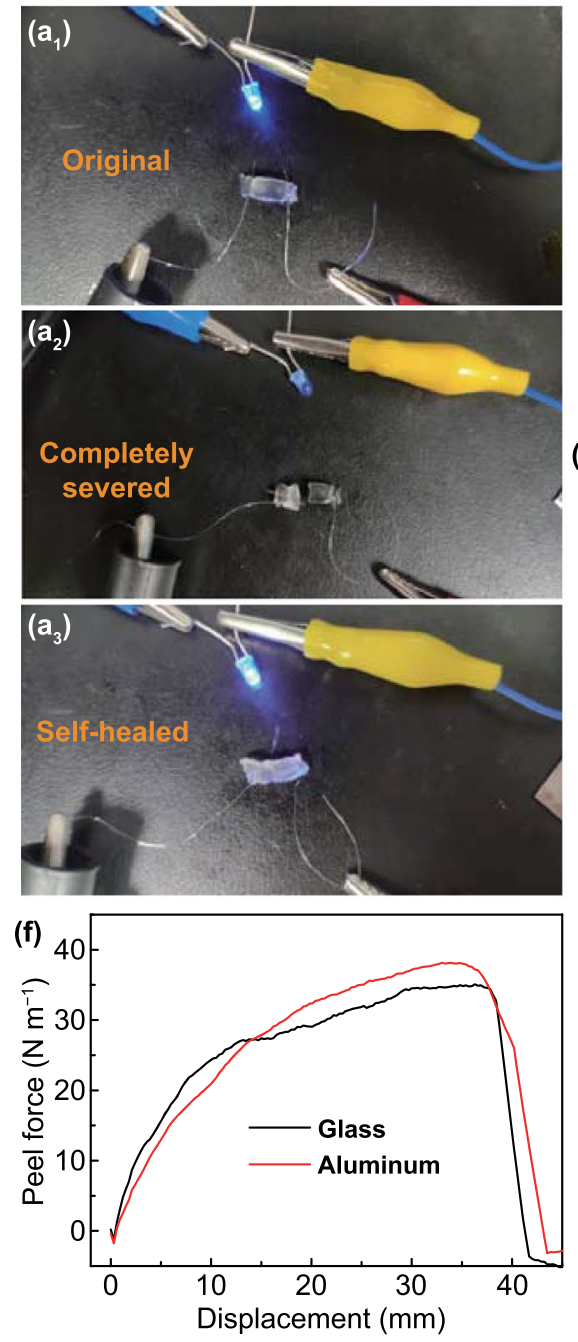

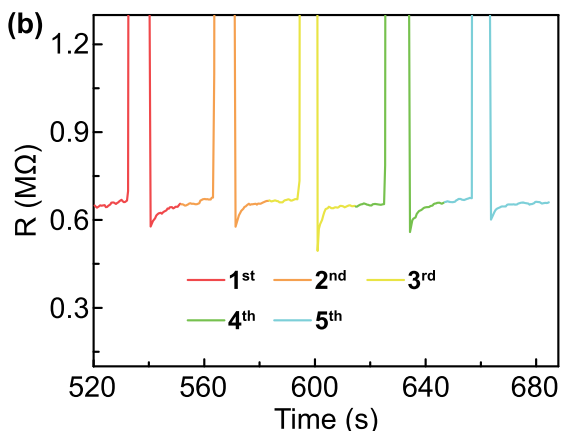

(d)
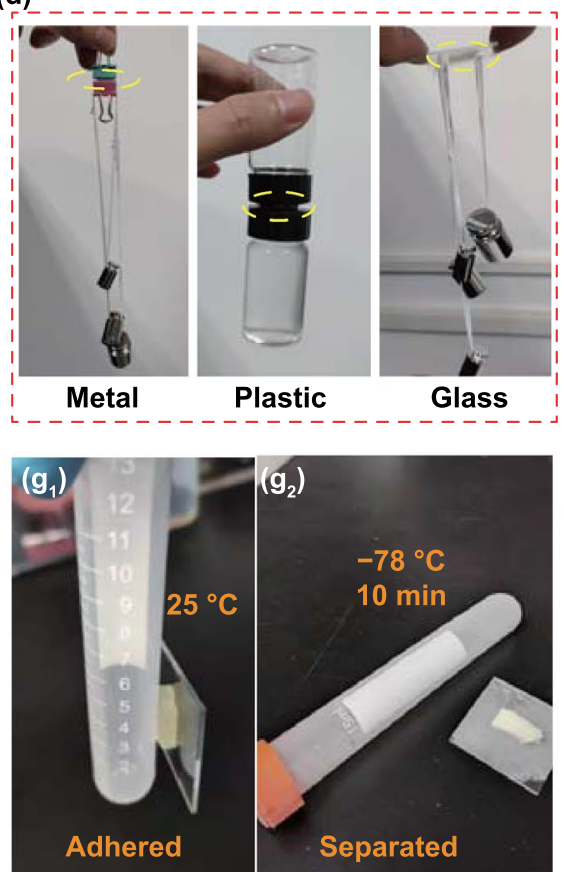

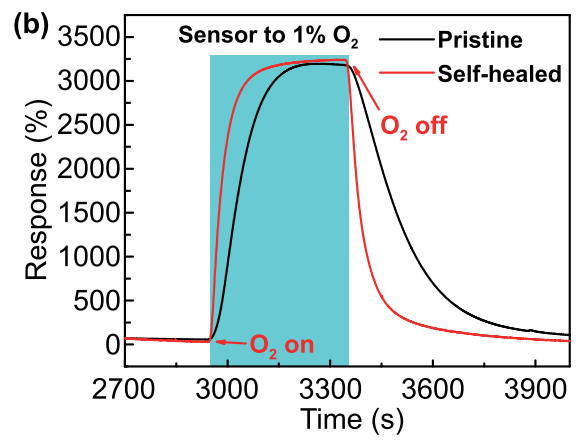

(e)
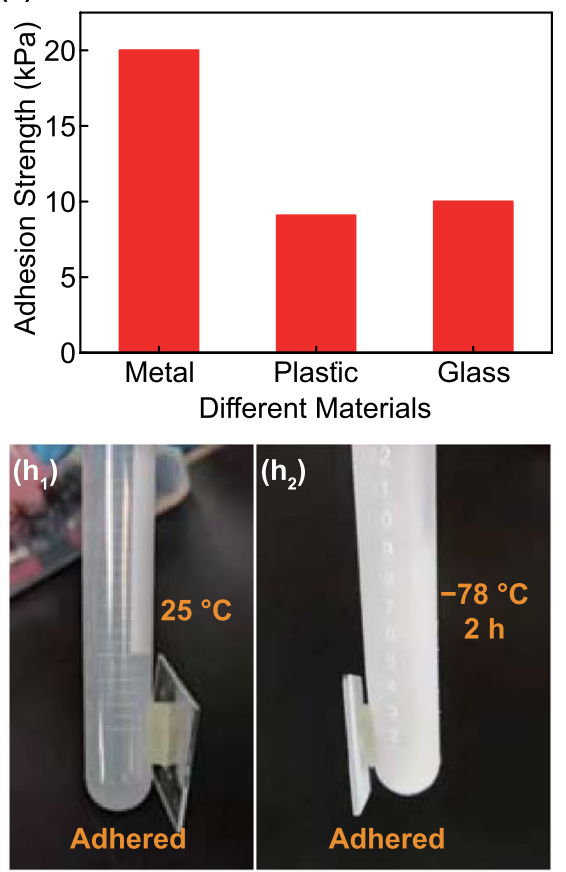

Fig. 3 Self-healing and self-adhesive abilities of organohydrogel. a The change of on and off states of the LED indicator in the series circuit when the organohydrogel was in the states of (1) original, (2) completely severed, and (3) self-healed. b Time evolution of cyclical electrical self-healing processes of organohydrogels by real-time resistance measurement. c Dynamic responses of the sensor to $1 \% \mathrm{O}_{2}$ in the pristine and self-healed states. d Photographs showing the organohydrogel (marked by the yellow circle) adhered firmly to various materials: metals, plastic, and glass. e The adhesion strength of organohydrogel on different substrates. $\mathbf{f}$ Peel force versus displacement curves of organohydrogel when adhered on different substrates. Photographs depicting $\mathbf{g}$ the untreated hydrogel could and could not adhere to the plastic centrifuge tube at 25 and $-78{ }^{\circ} \mathrm{C}$, respectively, whereas $\mathbf{h}$ the organohydrogel adhered firmly to the plastic centrifuge tube at both temperatures 
the resistance of the organohydrogel before and after the disconnection and self-healing (Fig. 3b). It was found that the resistance of the organohydrogel returned quickly to the original level after self-healing, illustrating that the repeated disconnection and reconnection of the organohydrogel at RT did not impair the electrical conductivity, reflecting the high self-healing efficiency. It is because the disconnected organohydrogel can utilize many dynamic hydrogen bonds formed between hydroxyl and amino groups on the chitosan chain and the PAM chain to reconnect the polymer chains, which helps restore its conductivity and some mechanical properties. To further test the self-healability in the gas sensing properties, we cut off the organohydrogel and then contacted the cuts to make it heal naturally. The response of the organohydrogel sensor to $1 \% \mathrm{O}_{2}$ after self-healing was about $3170 \%$, which was basically the same as that before disconnection (3200\%) (Fig. 3c). To better recover its mechanical properties, after the cuts of the broken organohydrogel were brought to contact with each other, they were wrapped with a tin foil and then heated at $95{ }^{\circ} \mathrm{C}$ for $10 \mathrm{~min}$. The organohydrogel repaired by the heating method could still be stretched to $250 \%$ strain without the appearance of obvious gaps, while the naturally repaired organohydrogel would break again when it was subjected to about $100 \%$ strain (Fig. S8a-b). Moreover, the elastic modulus of the organohydrogel repaired by heating is $289 \mathrm{kPa}$, which is higher than that of the natural repaired counterpart $(170 \mathrm{kPa})$. The increased healing efficiency after heating may be related to the accelerated movement of polymer chains, which promotes the reconnection of polymer chains. The readily self-healed electrical, gas sensing, and mechanical properties of the organohydrogels are conducive to long-term and durable wearable applications $[55,56]$.

The self-adhesive properties enable hydrogels to be directly adhered to various materials without requiring external adhesives, which is essential for a number of emerging applications, such as wearable sensors and bioelectronics, although self-adhesive gas sensor has seldom been reported [57]. Hydrogels generally achieve strong adhesion through the synergy of chemical bonds, the topology of connection, and mechanics of dissipation $[58,59]$. Note that chitosan contains numerous amino and hydroxyl groups. Furthermore, both PAM and 1,2-PD contain abundant hydroxyl groups. Therefore, the PAM-CS DN organohydrogel is a strong hydrogen-bonded complex with excellent self-adhesive ability on different materials [60]. As shown in Fig. 3d, two metal clips were firmly glued together by the organohydrogel, and the lower metal clip could bear a weight of $80 \mathrm{~g}$. Furthermore, two plastic bottle caps could also be glued together by the organohydrogel. The glass bottle below was filled with water and weighed $40 \mathrm{~g}$ in total. In addition, two glass slides were glued by the organohydrogel, and the lower glass sheet could withstand a weight of $100 \mathrm{~g}$. Notably, the organohydrogel displays varied adhesion strength on different materials. Specifically, the adhesion strengths on glass, plastic, and metal (aluminum) were 20, 9.1, and $10 \mathrm{kPa}$, respectively (Fig. 3e). To further evaluate the adhesive properties, the peel strength of organohydrogel to various substrates was measured using the $180^{\circ}$ peeling experiment (Fig. S9a). Figure 3f shows the peel force vs displacement curves of organohydrogel on glass and aluminum sheets, from which the peel strength of organohydrogel on both glass and aluminum sheets was determined as $35 \mathrm{~N} \mathrm{~m}^{-1}$.

Although both the hydrogel and organohydrogel exhibited good adhesion at RT, the organohydrogel with a water-diol binary solvent displayed much better self-adhesive ability at low temperatures, e.g., $-78^{\circ} \mathrm{C}$ due to its excellent anti-frost capacity. After the untreated hydrogel and organohydrogel had adhered to the plastic centrifuge tube, they were stored in a low-temperature box $\left(-78^{\circ} \mathrm{C}\right)$. It was found that the untreated hydrogel completely froze into ice and thus separated from the tube after $10 \mathrm{~min}$, while the organohydrogel showed no signs of freezing after $2 \mathrm{~h}$ and therefore could still firmly adhere to the tube (Fig. $3 \mathrm{~g}$, h). It is because the untreated hydrogel has a freezing point of $-16^{\circ} \mathrm{C}$, and a large part of the water molecules in the solvent freezes at a low temperature, causing the phase separation between polymers and solvents [61]. While the freezing point of the organohydrogel is below $-120^{\circ} \mathrm{C}$, so there will be no phase separation between the polymer and the solvent. At this time, numerous hydrogen bonds inside can still function normally at a low temperature of $-78{ }^{\circ} \mathrm{C}$, making the organohydrogel maintain excellent self-adhesiveness. These results collectively demonstrate the excellent self-adhesive properties of DN organohydrogel within a wide range of temperatures, which allows the organohydrogel sensor to be directly attached to people's clothing to monitor the ambient oxygen concentration without the demand for additional adhesive items, such as tape (Fig. S9b). Even when the clothing was highly deformed, no detachment between the sensor and the clothing was observed, manifesting the great promise of the adhesive sensor for wearable application. 


\subsection{Gas Sensing Properties under Different Deformations, Humidity, and Temperatures}

Compared with traditional $\mathrm{O}_{2}$ sensors, this organohydrogel $\mathrm{O}_{2}$ sensor provides the unique capability to work under various mechanical deformations, such as stretching and bending (Fig. 4). Furthermore, such large deformation as $100 \%$ tensile strain did not degrade the gas sensing performance, but rather improved both the sensitivity and response/recovery speeds. Specifically, the greater the tensile strain applied to the sensor, the higher its response to oxygen, and meanwhile the shorter the response and recovery time. Also, when the $100 \%$ tensile strain was applied to the sensor, the response of the sensor to $1 \%$ $\mathrm{O}_{2}$ increased from 3210 to $4041 \%$, suggesting an increment of $26 \%$. The response and recovery time of the sensor were reduced from 194 and $221 \mathrm{~s}$ to 128 and $172 \mathrm{~s}$, respectively, depicting a reduction of $34 \%$ and $22 \%$, respectively (Fig. 4a-c). Possibly this is because the baseline of response curve before exposure to oxygen was declined due to the increased resistance of organohydrogel with strain (Fig. S10), whereas the electrons produced or consumed at the hydrogel-electrode interface due to electrochemical reactions were unchanged, leading to the
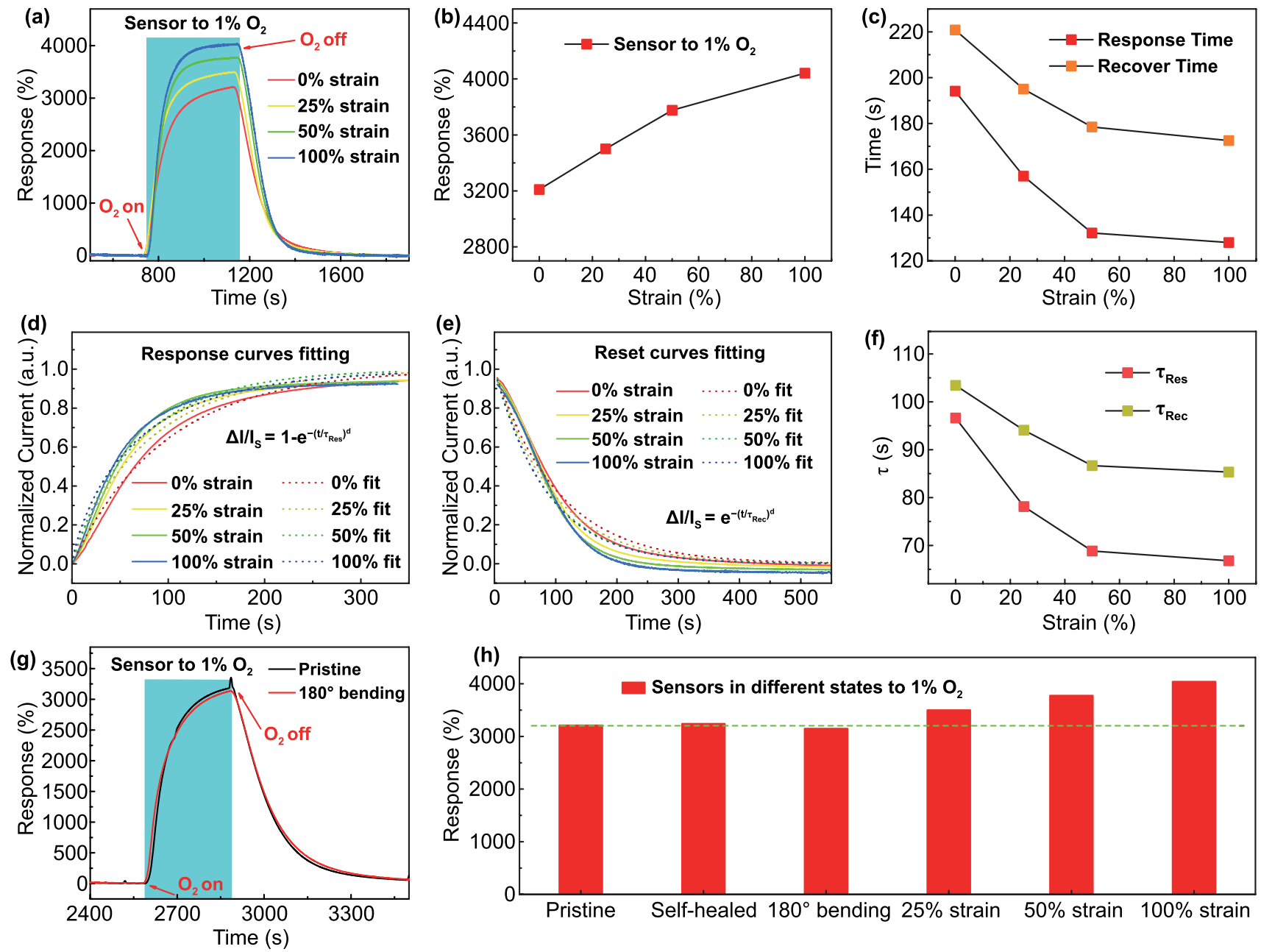

Fig. 4 Influence of deformation on $\mathrm{O}_{2}$ sensing properties of organohydrogel sensor. a Dynamic response curves to $1 \% \mathrm{O}_{2}$ at $0 \%, 25 \%, 50 \%$, and $100 \%$ tensile strains. b Response extracted from a versus strain. c Response and recovery time $t_{90}$ extracted from a versus strain. d Normalized current variations in response to $1 \% \mathrm{O}_{2}$ at different strains with fits to the exponential growth formula. e Normalized current variations in the recovery process with fits to the exponential decay formula at different strains. f Plots of time constants $\left(\tau_{\operatorname{Res}}\right.$ and $\left.\tau_{R e c}\right)$ versus tensile strain. $\mathbf{g}$ Real-time responses to $1 \% \mathrm{O}_{2}$ in pristine and $180^{\circ}$ bending states. $\mathbf{h}$ Quantitative responses to $1 \% \mathrm{O}_{2}$ in the states of pristine, self-healed, $180^{\circ}$ bending, $25 \%, 50 \%$, and $100 \%$ tensile strains 
increased relative current variation (response). Furthermore, both the internal structure and the surface area of the organohydrogel were changed during stretching. Specifically, the curly polymer chains straightened, and the pores in the organohydrogel might become larger, which promoted the diffusion of oxygen into the organohydrogel. In addition, the surface area of organohydrogel increased with strain, enhancing the adsorption of oxygen. Different from the freestanding organohydrogel as used for the gas sensing in Fig. 2c, the stretched organohydrogel was attached on a glass slide for studying the impact of strain on the gas sensing characteristics (Fig. S10a). Since the gas adsorption at the organohydrogel-glass interface was blocked in this case, it took a longer time to complete the gas diffusion and reaction.

To further research on the change of response/recovery time with strain, the dynamic response curves of the sensor under different tensile strains were fitted according to the exponential growth formula $[62,63]$ :

$\Delta I / I_{S}=1-e^{-\left(t / \tau_{\text {Res }}\right)^{d}}$

where $I_{S}$ is the saturation current, $\tau_{\text {Res }}$ is the response time constant, $d$ is an exponent between 0 and 1 . Figure $4 \mathrm{~d}$ shows the experimental result and fitting curves at different strains. From the exponential fitting, the response time constants $\tau_{\text {Res }}$ of $96.6,78.1,68.8$, and $66.8 \mathrm{~s}$ were obtained at $0 \%$, $25 \%, 50 \%$, and $100 \%$ strains, respectively, with the exponent $d$ of $0.99,0.92,0.93$, and 0.82 , respectively. Similarly, the dynamic reset curves were fitted according to the exponential decay formula:

$\Delta I / I_{S}=e^{-\left(t / \tau_{\mathrm{ReC}}\right)^{d}}$

where $\tau_{R e c}$ is the recovery time constant, which is 103.4 , $94.1,86.7$, and $85.3 \mathrm{~s}$ at $0 \%, 25 \%, 50 \%$, and $100 \%$ strains, respectively (Fig. 4e). It is clear that the greater the tensile strain, the smaller the $\tau_{R e s}$ and $\tau_{R e c}$ (Fig.4f), which is consistent with the change of response and recovery time with strain as illustrated in Fig. 4c.

The stretchable organohydrogel sensor also displayed the clear electromechanical response, which was the change of resistance with tensile strain. It can be seen that the resistance augmented linearly with tensile strain from 0 to $200 \%$, from which the gauge factor (GF) of 3.24 was derived (Fig. S10b-c). This suggests the possibility of employing the organohydrogel sensor to detect multiple stimuli. The remarkable electromechanical response of the sensor is attributed to the geometric variation of organohydrogel. As long as the response of the oxygen sensor in various tensile states is tested in advance, the interference of tensile strain to gas sensing can also be eliminated through the calibration with an external strain sensor in the practical wearable application. Except for tensile strain, other deformations such as bending and twisting hardly affect the resistance and response of the sensor. Notably, the excellent self-adhesive properties of the organohydrogel enabled the sensor to be bent for $180^{\circ}$ and directly stuck on the glass slide to investigate its gas sensing characteristics at a highly bent state without using external adhesive (Fig. S11). Figure $4 \mathrm{~g}$ shows that the response of the sensor to $1 \% \mathrm{O}_{2}$ before and after bending was basically the same, i.e., $3200 \%$. Therefore, the response of the sensor was maintained whether it was deformed or after being broken and self-healed naturally (Fig. 4h). These attributes not only make the sensor very suitable for the application in wearable electronics but also extend its life span [64].

Notably, both humidity and temperature are important influencing factors for room-temperature gas sensors. In actual wearable applications, the humidity and temperature of the environment where different people are located are different, which requires the organohydrogel oxygen sensor to work normally under various humidity and temperatures. Figure $5 \mathrm{a}, \mathrm{b}$ shows the responsive behavior of the sensor to $1 \% \mathrm{O}_{2}$ under different RH. Obviously, when the RH is about $52.5 \%$, the sensor displays the highest response to oxygen, which is $4538 \%$. When the $\mathrm{RH}$ is higher or lower than $52.5 \%$, the response shows a downward trend. It is because the content of water molecules in the environment is overly low when the $\mathrm{RH}$ is low, which is unfavorable to the progress of the reduction reaction at the cathode, resulting in a decrease in the redox response. However, the organohydrogel absorbs superabundant water molecules by itself when the RH is high, thereby increasing its conductance and raising the baseline of the response curve before exposure to oxygen. This becomes the main factor affecting the response, leading to a decrease in the relative current variations. In addition, the response and recovery time of the sensor will decrease as the RH increases, which dropped from $58.7 \mathrm{~s}$ and $102.3 \mathrm{~s}$ under $11.3 \% \mathrm{RH}$ to $28.4 \mathrm{~s}$ and $55 \mathrm{~s}$ under $90.5 \% \mathrm{RH}$, respectively (Fig. $5 \mathrm{c}$ ). This is also due to the increase in the content of water molecules in the environment, which increases the rate of redox reactions. Similar to the study of the influence of tensile strain on the 

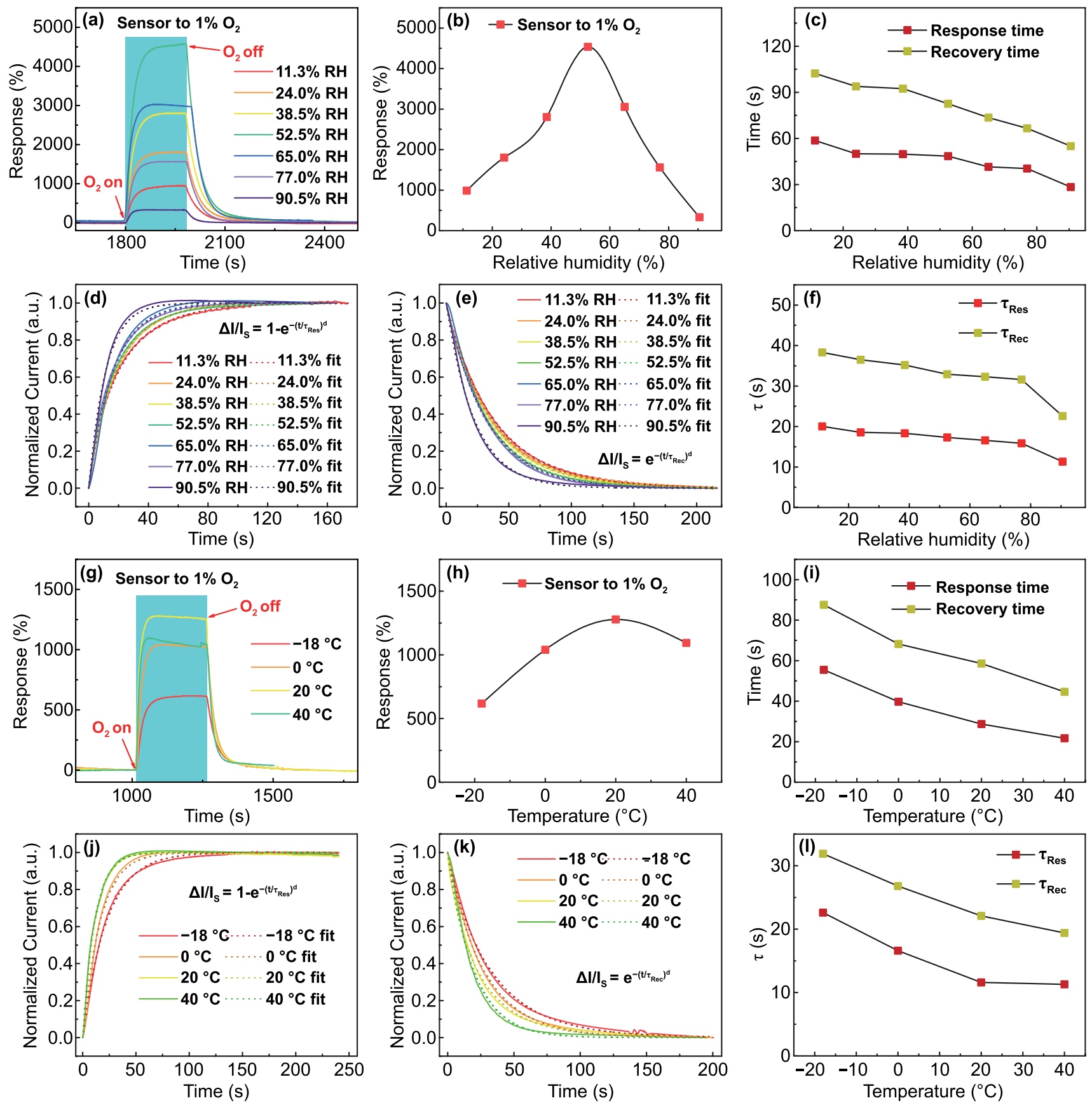

Fig. 5 Influences of humidity and temperature on $\mathrm{O}_{2}$ sensing properties of organohydrogel sensor. a Dynamic response curves to $1 \%$ Onder $11.3 \%, 24.0 \%, 38.5 \%, 52.5 \%, 65.0 \%, 77.0 \%$, and $90.5 \%$ RH. b Response extracted from a versus relative humidity. c Response and recovery time $\mathrm{t}_{90}$ extracted from a versus relative humidity. $\mathbf{d}$ and e Normalized current variations in response to $1 \% \mathrm{O}_{2}$ under different RH with fits to the exponential growth formula in the response and recovery processes, respectively. $\mathbf{f}$ Plots of time constants $\left(\tau_{R e s}\right.$ and $\left.\tau_{R e c}\right)$ versus relative humidity. $\mathbf{g}$ Dynamic response curves to $1 \% \mathrm{O}_{2}$ at $-18,0,20$, and $40^{\circ} \mathrm{C}$. $\mathbf{h}$ Response extracted from $\mathbf{g}$ versus temperature. $\mathbf{i}$ Response and recovery time $t_{90}$ extracted from $\mathbf{g}$ versus temperature. $\mathbf{j}$ and $\mathbf{k}$ Normalized current variations in response to $1 \% \mathrm{O}_{2}$ under different temperatures with fits to the exponential growth formula in the response and recovery processes, respectively. I Plots of time constants $\left(\tau_{R e s}\right.$ and $\left.\tau_{R e c}\right)$ versus temperature

response/recovery speeds, the dynamic response and reset curves of the sensor under different RH were fitted according to the exponential growth formula, and $\tau_{R e s}$ and $\tau_{R e c}$ were extracted (Fig. 5d-f). Clearly, with the increase in RH, $\tau_{R e s}$ and $\tau_{R e c}$ decrease from 20 and $38.3 \mathrm{~s}$ under $11.3 \% \mathrm{RH}$ to $11.3 \mathrm{~s}$ and $22.6 \mathrm{~s}$ under $90.5 \% \mathrm{RH}$, respectively, which 
are the same as the changing trend of response and recovery time in Fig. 5c.

In addition, the responsive behavior of the sensor to $1 \%$ $\mathrm{O}_{2}$ at different temperatures was investigated. The result in Fig. 5g-h shows that the sensor has an optimal working temperature, which is about $20^{\circ} \mathrm{C}$. When the temperature is higher or lower than $20^{\circ} \mathrm{C}$, the response will also show a downward trend. The low response at $-18{ }^{\circ} \mathrm{C}$ is because the low temperature is unfavorable for the progress of electrochemical reactions [65]. However, when the sensor is at a high temperature, the baseline of the response curve will also raise due to the thermally activated ionic migration, which becomes the main factor affecting the response, resulting in a decrease in the relative current variation [66]. As with humidity, the response and recovery time of the sensor will decrease with increasing temperature, which dropped from 55.5 and $87.6 \mathrm{~s}$ at $-18{ }^{\circ} \mathrm{C}$ to $21.7 \mathrm{~s}$ and $44.6 \mathrm{~s}$ at $40{ }^{\circ} \mathrm{C}$, respectively (Fig. 5i). This is because the rise in temperature increases the rate of electrochemical reactions, which in turn increases the response/recovery speeds. Fitting the response and reset curves with the exponential decay formula allows for the extraction of the $\tau_{R e s}$ and $\tau_{\text {Rec }}$ (Fig. 5j, k). Their tendency to change with temperature is the same as that of the response and recovery time, which decreases from 22.6 and $31.9 \mathrm{~s}$ at $-18{ }^{\circ} \mathrm{C}$ to $11.3 \mathrm{~s}$ and $19.4 \mathrm{~s}$ at $40{ }^{\circ} \mathrm{C}$ (Fig. 51).

Therefore, the best working temperature and humidity of the organohydrogel oxygen sensor are $20{ }^{\circ} \mathrm{C}$ and $52.5 \% \mathrm{RH}$, respectively, which is the comfort zone in our daily life. Note that the sensor can also work normally no matter in low/high temperature, dry/wet environments, or under different tensile strains. Just like eliminating the interference of tensile strain on the sensor, calibrating the organohydrogel oxygen sensor by integrating temperature and humidity sensors can also eliminate the interference of them. The capability to work normally within a broad range of temperature and humidity not only greatly expands the application scope of the oxygen sensor, but also makes it applicable to wearable fields and increases the value of its practical application.

\subsection{Investigation of the Sensing Mechanism}

To confirm the aforementioned electrochemical gas sensing mechanism of organohydrogel sensor (Fig. 1h), we designed a series of experiments. First, we exploited SEM and its energy dispersive spectroscopy (EDS) module to investigate the variations of surface morphology and composition of electrodes before and after the gas sensing test using a high DC voltage $(5 \mathrm{~V})$. Two pieces of organohydrogel prepared in the same batch were connected with $\mathrm{Ag}$ wires, which functioned as the electrodes of the sensors when the DC voltage was and was not applied in the experimental and control groups, respectively, for $5 \mathrm{~h}$. Both of the two sensors were exposed to the air (with $20.9 \% \mathrm{O}_{2}$ ). After $5 \mathrm{~h}$, the surface morphology and composition of anode and cathode materials (Ag wires) in the experimental and control groups were compared (Figs. 6a, S12, and Table 1). For the experimental group, the anode $\mathrm{Ag}$ was oxidized to form $\mathrm{AgCl}$, while the cathode Ag kept intact. It verified that oxygen was reduced at the cathode, while the cathode metal itself was not consumed in the reaction. For the control group, neither the morphology nor the composition variation was observed for both the anode and cathode materials. It confirms the aforementioned redox reaction-based gas sensing mechanism.

Note that we have also specially devised a cathode encapsulation experiment to further certify the proposed sensing mechanism. Specifically, the response of the sensor to $1 \% \mathrm{O}_{2}$ was compared before and after the cathode was encapsulated by an Ecoflex film with enough thickness to isolate it from outside air (Fig. 6b). Before the Ecoflex precursor solidified, the cathode of the sensor was immersed in it. After the polymerization of Ecoflex, the cathode of the sensor was naturally encapsulated (Fig. S13). The anode of the sensor was normally exposed during the sensing test. Figure $6 \mathrm{c}$ shows that the response of the sensor to the same concentration of oxygen dropped significantly, close to 0 , when the cathode was encapsulated, manifesting that the oxygen reacted at the cathode-organohydrogel interface. This also explains the phenomenon of positive current displacement of the sensor in response to oxygen.

To further explore the redox reactions occurring at the interface, the cyclic voltammetry (CV) curves of the sensor under $\mathrm{N}_{2}$ and air environment were scanned when $\mathrm{Ag}$ and carbon electrodes were applied. It can be seen from Fig. $6 \mathrm{~d}$ that the $\mathrm{CV}$ curves measured by the carbon electrodes in both $\mathrm{N}_{2}$ and air environment basically coincided, implying that no redox peak appeared on the CV curves. This is because the carbon electrodes cannot be oxidized by $\mathrm{O}_{2}$, and thus the sensor cannot undergo an electrochemical reaction, demonstrating the important role of electrodes in the gas sensing of ion-conducting organohydrogel. The sensor 

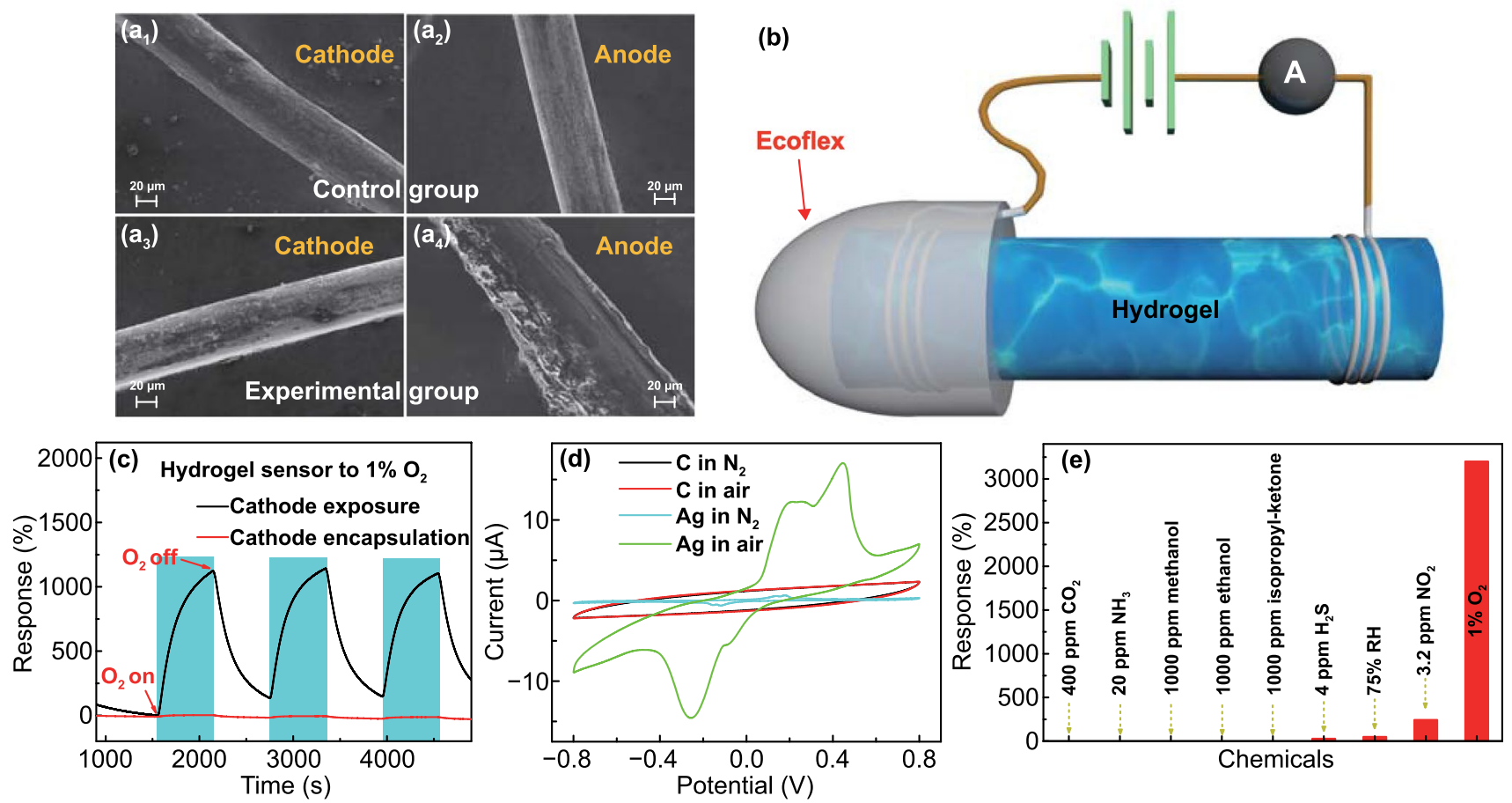

Fig. 6 a SEM images of (1) cathode and (2) anode in the control group, and (3) cathode and (4) anode in the experimental group after exposure to air for $5 \mathrm{~h}$. b Schematic illustrating the device configuration in the cathode encapsulation experiment. $\mathbf{c}$ Dynamic responses of the sensor to $1 \% \mathrm{O}_{2}$ before and after encapsulation of cathode. $\mathbf{d}$ Current-Potential curves of the sensor when $\mathrm{Ag}$ and carbon (C) electrodes were employed in $\mathrm{N}_{2}$ and air, respectively. e Comparison in the responses of the sensor to $400 \mathrm{ppm} \mathrm{CO}_{2}, 20 \mathrm{ppm} \mathrm{NH}_{3}, 4 \mathrm{ppm} \mathrm{H}_{2} \mathrm{~S}, 3.2 \mathrm{ppm} \mathrm{NO}_{2}$, and $1 \% \mathrm{O}_{2}$

Table 1 EDS element analysis of the cathode and anode in the control and experimental groups after exposure to $\mathrm{O}_{2}$ for $5 \mathrm{~h}$

\begin{tabular}{llll}
\hline Groups (\%) & Electrode & Element & $\begin{array}{l}\text { Atomic } \\
\text { percent- } \\
\text { age }\end{array}$ \\
\hline Control & Cathode & $\mathrm{Ag}$ & 100 \\
& Anode & $\mathrm{Ag}$ & 100 \\
Experimental & Cathode & $\mathrm{Ag}$ & 100 \\
& Anode & $\mathrm{Ag}$ & 39.43 \\
& & $\mathrm{Cl}$ & 46.4 \\
& & $\mathrm{Na}$ & 14.17 \\
\hline
\end{tabular}

based on Ag electrodes exhibited only two very small redox peaks (caused by the redox reactions of trace $\mathrm{O}_{2}$ dissolved in organohydrogel) in a $\mathrm{N}_{2}$ environment, while two obvious redox peaks appeared in air (resulting from the oxidation of Ag electrode by $\mathrm{O}_{2}$ ). This experiment consolidated the electrochemical reaction-based gas sensing mechanism from another perspective.

The internal three-dimensional polymer network structure of PAM-CS hydrogel allows it to retain a large amount of water in a solid form, and both the hydroxyl and the amino groups of chitosan can form hydrogen bonds with $\mathrm{H}_{2} \mathrm{O}$ and $\mathrm{O}_{2}$ to promote their adsorption. All of these provide favorable conditions for the full progress of electrochemical reactions. In addition, the incorporation of 1,2-PD in the organohydrogel brings a large number of hydroxyl groups, which can also form hydrogen bonds with $\mathrm{H}_{2} \mathrm{O}$ and $\mathrm{O}_{2}$. It not only improves the moisture retention of organohydrogel but also promotes the adsorption of reactants, which is conducive to the proceeding of electrochemical reactions. Figure S14 shows the response of the organohydrogel sensor to $1 \% \mathrm{O}_{2}$ is significantly greater than that of the hydrogel sensor, which may stem from the lower current baseline of the organohydrogel sensor and the enhanced ability of organohydrogel to adsorb both $\mathrm{H}_{2} \mathrm{O}$ and $\mathrm{O}_{2}$ by readily forming hydrogen bonds between these molecules and 1,2-PD. Apart from $\mathrm{O}_{2}$, the sensor showed negligible responses to other oxidizing or reducing gases or other gaseous chemicals, such as $\mathrm{CO}_{2}$, $\mathrm{NH}_{3}$, methanol, ethanol, isopropyl-ketone, $\mathrm{H}_{2} \mathrm{~S}$, humidity, and $\mathrm{NO}_{2}$ (Figs. 6e and S15). For instance, the response of the sensor to $4 \mathrm{ppm} \mathrm{H}_{2} \mathrm{~S}$ is only $29 \%$, which is two orders of magnitude lower than that to $1 \% \mathrm{O}_{2}$. Although its response 
to $3.2 \mathrm{ppm} \mathrm{NO}_{2}$ can reach $240 \%$, the sensor cannot detect the ppm level of $\mathrm{NO}_{2}$ in an air environment, owing to the high response and high concentration of oxygen (Fig. S16). Moreover, the content of these gases in the common environment is extremely low compared with that of $\mathrm{O}_{2}$. Therefore, the interference from these gases is negligible, reflecting the high selectivity of the $\mathrm{O}_{2}$ sensor.

\subsection{Practical Applications}

Finally, we have proved the feasibility of applying the $\mathrm{O}_{2}$ sensor for real-life applications, such as the real-time detection of $\mathrm{O}_{2}$ concentration in human exhaled breath. We liquid-sealed the sensor with two plastic bottles filled with water at the front and back and then exploited the gas distribution system to feed $\mathrm{N}_{2}$ into the bottle where the sensor was located. Then, $20.9 \% \mathrm{O}_{2}$, the gas exhaled by humans, $16 \%$ and $14 \% \mathrm{O}_{2}$ were sequentially introduced into the bottle with a $\mathrm{N}_{2}$ background (Fig. 7a). Figure $7 \mathrm{~b}$ shows that the Faraday current generated by human breath is between that produced by $16 \%$ and $14 \% \mathrm{O}_{2}$, suggesting that the $\mathrm{O}_{2}$ content of the gas exhaled by the volunteer is about $15 \%$, which is in line with the reality [67]. Considering the portability of the device, it is only necessary to replace the liquid sealing device with a small air bag to make it a portable system for monitoring human breath in practical application. It is worth noting that we have also deployed this $\mathrm{O}_{2}$ sensor to detect human breath directly in the air. To preclude the influence of humidity on $\mathrm{O}_{2}$ sensing, the sensor was simply covered by a sheet of hydrophobic Ecoflex to prevent the condensation of water on the organohydrogel (Fig. S17). Normally, the $\mathrm{O}_{2}$ concentration from exhaled breath $(\sim 15 \%)$ is lower than that in the air $(\sim 21 \%)$. Thereby, the $\mathrm{O}_{2}$ concentration around the sensor decreased when blowing the sensor with the mouth. Therefore, the current flowing through the sensor showed a downward trend. After stopping blowing, the

(a)
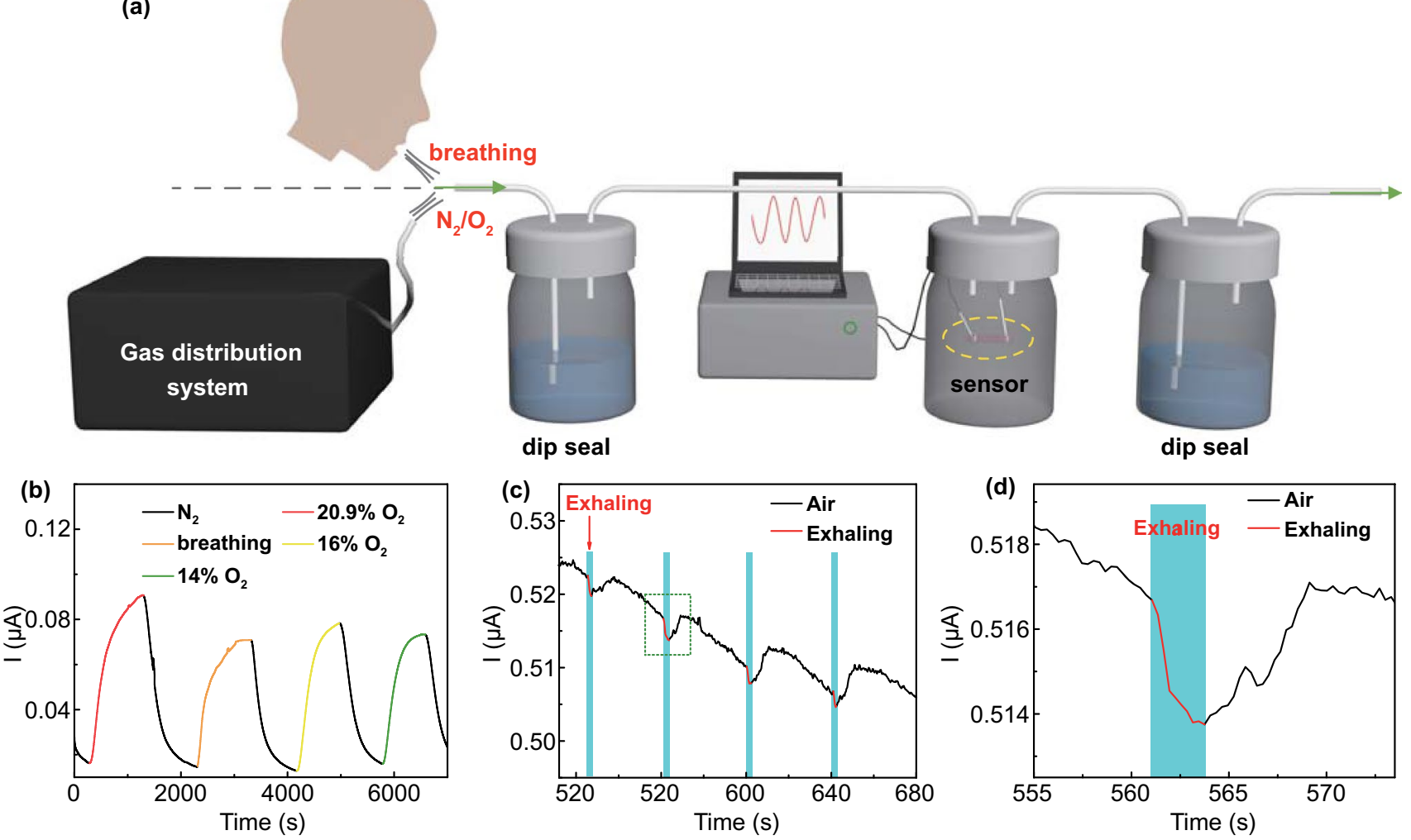

Fig. 7 Application of the sensor in human respiration monitoring. a Schematic illustrating the setup for real-time detection of $\mathrm{O}_{2}$ concentration in human exhaled breath. b Dynamic response to $20.9 \% \mathrm{O}_{2}$, exhaled breath, $16 \%$, and $14 \% \mathrm{O}_{2}$. $\mathbf{c}$ Real-time response to the gas exhaled by the human in the air. $\mathbf{d}$ Magnified image of the green box in $\mathbf{c}$ 
$\mathrm{O}_{2}$ concentration around the sensor resumed, leading to the recovery of current to the original level (Fig. 7c, d). All these results manifest that this $\mathrm{O}_{2}$ sensor can be exploited to monitor human breath in real time.

Note that chitosan has been widely used in wound dressings because of its outstanding biocompatibility, biodegradation, antibacterial, and endogenous hemostasis properties [68]. The hydrophilic and bio-inert nature of PAM make it attractive for biomedical applications, among others [69]. Notably, appropriate $\mathrm{O}_{2}$ concentration in the wound tissue is essential for promoting wound healing [70]. Combining these characteristics and the selfadhesive property of the PAM-CS DN organohydrogel sensor, it can be potentially attached to the wound, not only for monitoring the $\mathrm{O}_{2}$ concentration at the wound in real-time but also for promoting the wound healing simultaneously. These advantages and application scenarios render the $\mathrm{O}_{2}$ sensor promising for wearable healthcare monitoring.

\section{Conclusions}

In summary, we have successfully fabricated a stretchable, self-healable, self-adhesive, and high-performance $\mathrm{O}_{2}$ sensor based on PAM-CS DN organohydrogel, which was prepared through a facile soaking and solvent replacement strategy. Compared with the pristine hydrogel, the organohydrogel displays greatly enhanced mechanical strength, moisture retention, freezing resistance, and $\mathrm{O}_{2}$ sensitivity. The DN organohydrogel-based $\mathrm{O}_{2}$ sensor provides a full concentration detection range (0-100\%), low LOD (5.7 ppm), sensitivity of $0.2 \% / \mathrm{ppm}$, linearity, selectivity, repeatability at RT, and the capability to work normally under a broad range of humidity and temperatures, which are competitive in comparison with that of existing $\mathrm{O}_{2}$ sensors. Attributing to the impressive deformability of organohydrogel, the sensors kept working under large deformations, such as $100 \%$ tensile strain, without degrading the sensing performance. Furthermore, both the sensitivity and the response/recovery speeds are boosted by applying strain. Notably, all the gas sensitivity, electrical conductance, and mechanical deformability of the organohydrogel are readily self-healed after being broken by an external force. In addition, due to the excellent self-adhesive property of organohydrogel within a broad range of temperatures, the $\mathrm{O}_{2}$ sensor can be directly adhered to various irregular substrates for working without demanding additional tapes. These unique attributes promote the development and application of the sensor in the field of wearable electronics.

Importantly, we have proposed the electrochemical reaction mechanism at the hydrogel-electrode interface to understand the $\mathrm{O}_{2}$ sensing behaviors of ion-conducting organohydrogel. This sensing mechanism was further corroborated via specially designed experiments. The practical applicability of this sensor in monitoring the $\mathrm{O}_{2}$ concentration from exhaled breath was also verified. Despite that only one type of organohydrogel was investigated here, its performance already exceeded that of conventional $\mathrm{O}_{2}$ transducing materials. This work opens the door to fabricating stretchable, self-healable, self-adhesive $\mathrm{O}_{2}$ sensors with high performance at RT using a family of ion-conducting organohydrogels as novel sensing materials for emerging wearable and healthcare applications.

Acknowledgements J.W. acknowledges financial support from the National Natural Science Foundation of China (61801525), the Guangdong Basic and Applied Basic Research Foundation (2020A1515010693), the Guangdong Natural Science Funds Grant (2018A030313400), the Science and Technology Program of Guangzhou (201904010456), and the Fundamental Research Funds for the Central Universities, Sun Yat-sen University (2021qntd09).

Funding Open access funding provided by Shanghai Jiao Tong University.

Open Access This article is licensed under a Creative Commons Attribution 4.0 International License, which permits use, sharing, adaptation, distribution and reproduction in any medium or format, as long as you give appropriate credit to the original author(s) and the source, provide a link to the Creative Commons licence, and indicate if changes were made. The images or other third party material in this article are included in the article's Creative Commons licence, unless indicated otherwise in a credit line to the material. If material is not included in the article's Creative Commons licence and your intended use is not permitted by statutory regulation or exceeds the permitted use, you will need to obtain permission directly from the copyright holder. To view a copy of this licence, visit http://creativecommons.org/licenses/by/4.0/.

Supplementary Information The online version contains supplementary material available at https://doi.org/10.1007/ s40820-021-00787-0. 


\section{References}

1. K. Xu, Y. Fujita, Y. Lu, S. Honda, M. Shiomi et al., A wearable body condition sensor system with wireless feedback alarm functions. Adv. Mater. 33(18), 2008701 (2021). https://doi. org/10.1002/adma.202008701

2. J. Wu, Y. Wei, H. Ding, Z. Wu, X. Yang et al., Green synthesis of 3D chemically functionalized graphene hydrogel for highperformance $\mathrm{NH}_{3}$ and $\mathrm{NO}_{2}$ detection at room temperature. ACS Appl. Mater. Interfaces 12(18), 20623-20632 (2020). https://doi.org/10.1021/acsami.0c00578

3. Z. Wu, L. Rong, J. Yang, Y. Wei, K. Tao et al., Ion-conductive hydrogel-based stretchable, self-healing, and transparent NO2 sensor with high sensitivity and selectivity at room temperature. Small (2021). https://doi.org/10.1002/smll.202104997

4. H. Yin, Y. Cao, B. Marelli, X. Zeng, A.J. Mason et al., Soil sensors and plant wearables for smart and precision agriculture. Adv. Mater. 33(20), 2007764 (2021). https://doi.org/10. 1002/adma.202007764

5. D. Yu, Z. Zheng, J. Liu, H. Xiao, G. Huangfu et al., Superflexible and lead-free piezoelectric nanogenerator as a highly sensitive self-powered sensor for human motion monitoring. Nano-Micro Lett. 13, 117 (2021). https://doi.org/10.1007/ s40820-021-00649-9

6. S.G. Chatterjee, S. Chatterjee, A.K. Ray, A.K. Chakraborty, Graphene-metal oxide nanohybrids for toxic gas sensor: a review. Sens. Actuators B 221, 1170-1181 (2015). https:// doi.org/10.1016/j.snb.2015.07.070

7. Z. Meng, R.M. Stolz, L. Mendecki, K.A. Mirica, Electricallytransduced chemical sensors based on two-dimensional nanomaterials. Chem. Rev. 119(1), 478-598 (2019). https://doi.org/ 10.1021/acs.chemrev.8b00311

8. T. Pham, G. Li, E. Bekyarova, M.E. Itkis, A. Mulchandani, $\mathrm{MoS}_{2}$-based optoelectronic gas sensor with sub-parts-per-billion limit of $\mathrm{NO}_{2}$ gas detection. ACS Nano 13(3), 3196-3205 (2019). https://doi.org/10.1021/acsnano.8b08778

9. Y. Wang, G. Duan, Y. Zhu, H. Zhang, Z. Xu et al., Room temperature $\mathrm{H}_{2} \mathrm{~S}$ gas sensing properties of $\operatorname{In}_{2} \mathrm{O}_{3}$ micro/nanostructured porous thin film and hydrolyzation-induced enhanced sensing mechanism. Sens. Actuators B 228, 74-84 (2016). https://doi.org/10.1016/j.snb.2016.01.002

10. R. Maughan, Carbohydrate metabolism. Surgery 27(1), 6-10 (2009). https://doi.org/10.1016/j.mpsur.2008.12.002

11. J.C. Downs, S.E. Conradi, C.A. Nichols, Suicide by environmental hypoxia (forced depletion of oxygen). Am. J. Forensic Med. Pathol. 15(3), 216-216 (1994). https://doi.org/10.1097/ 00000433-199409000-00008

12. Y.H. Kim, K.Y. Kim, Y.R. Choi, Y.S. Shim, J.M. Jeon et al., Ultrasensitive reversible oxygen sensing by using liquid-exfoliated $\mathrm{MoS}_{2}$ nanoparticles. J. Mater. Chem. A 4(16), 60706076 (2016). https://doi.org/10.1039/c6ta01277a

13. G. Hedenstierna, Oxygen and anesthesia: What lung do we deliver to the post-operative ward? Acta Anaesthesiol. Scand. 56(6), 675-685 (2012). https://doi.org/10.1111/j.1399-6576. 2012.02689.x
14. J. Ernsting, Breathing systems in aerospace. IEE Seminar. Low flow anaesthesia breathing systems-technology, safety and economics. London, UK (1999). https://doi.org/10.1049/ ic: 19990341

15. P. Wilmshurst, $\mathrm{ABC}$ of oxygen: diving and oxygen. BMJ 317, 996 (1998). https://doi.org/10.1136/bmj.317.7164.996

16. Y. Katayama, Y. Fujioka, K. Tsukada, Development of a patchtype flexible oxygen partial pressure sensor. IEEE J. Transl. Eng. Health. Med. 8, 1400607 (2020). https://doi.org/10.1109/ JTEHM.2020.3005477

17. B. Liu, H. Ni, D. Zhang, D. Wang, D. Fu et al., Ultrasensitive detection of protein with wide linear dynamic range based on core-shell SERS nanotags and photonic crystal beads. ACS Sens. 2(7), 1035-1043 (2017). https://doi.org/10.1021/acsse nsors.7b00310

18. H. Wang, S. Li, Y. Wang, H. Wang, X. Shen et al., Bioinspired fluffy fabric with in situ grown carbon nanotubes for ultrasensitive wearable airflow sensor. Adv. Mater. 32(11), 1908214 (2020). https://doi.org/10.1002/adma.201908214

19. M. Liao, P. Wan, J. Wen, M. Gong, X. Wu et al., Wearable, healable, and adhesive epidermal sensors assembled from mussel-inspired conductive hybrid hydrogel framework. Adv. Funct. Mater. 27(48), 1703852 (2017). https://doi.org/10.1002/ adfm.201703852

20. Q. Zhou, B. Ji, F. Hu, J. Luo, B. Zhou, Magnetized micropillar-enabled wearable sensors for touchless and intelligent information communication. Nano-Micro Lett. 13, 197 (2021). https://doi.org/10.1007/s40820-021-00720-5

21. K. Tao, Z. Chen, H. Yi, R. Zhang, Q. Shen et al., Hierarchical honeycomb-structured electret/triboelectric nanogenerator for biomechanical and morphing wing energy harvesting. Nano-Micro Lett. 13, 123 (2021). https://doi.org/10.1007/ s40820-021-00644-0

22. H. He, M. Zhang, T. Zhao, H. Zeng, L. Xing et al., A self-powered gas sensor based on PDMS/Ppy triboelectric-gas-sensing arrays for the real-time monitoring of automotive exhaust gas at room temperature. Sci. China Mater. 62, 1433-1444 (2019). https://doi.org/10.1007/s40843-019-9445-9

23. J.W. Kim, Y. Porte, K.Y. Ko, H. Kim, J.M. Myoung, Micropatternable double-faced $\mathrm{ZnO}$ nanoflowers for flexible gas sensor. ACS Appl. Mater. Interfaces 9(38), 32876-32886 (2017). https://doi.org/10.1021/acsami.7b09251

24. C. Liu, H. Tai, P. Zhang, Z. Yuan, X. Du et al., A high-performance flexible gas sensor based on self-assembled PANI$\mathrm{CeO}_{2}$ nanocomposite thin film for trace-level $\mathrm{NH}_{3}$ detection at room temperature. Sens. Actuators B 261, 587-597 (2018). https://doi.org/10.1016/j.snb.2017.12.022

25. E. Singh, M. Meyyappan, H.S. Nalwa, Flexible graphenebased wearable gas and chemical sensors. ACS Appl. Mater. Interfaces 9, 34544-34586 (2017). https://doi.org/10.1021/ acsami. $7 b 07063$

26. Y. Zhao, J.G. Song, G.H. Ryu, K.Y. Ko, W.J. Woo et al., Lowtemperature synthesis of $2 \mathrm{D} \mathrm{MoS}_{2}$ on a plastic substrate for a flexible gas sensor. Nanoscale 10(19), 9338-9345 (2018). https://doi.org/10.1039/c8nr00108a 
27. Y. Xu, J. Xie, Y. Zhang, F. Tian, C. Yang et al., Edge-enriched $\mathrm{WS}_{2}$ nanosheets on carbon nanofibers boosts $\mathrm{NO}_{2}$ detection at room temperature. J. Hazard. Mater. 411, 125120 (2021). https://doi.org/10.1016/j.jhazmat.2021.125120

28. Y. Xu, W. Zheng, X. Liu, L. Zhang, L. Zheng et al., Platinum single atoms on tin oxide ultrathin films for extremely sensitive gas detection. Mater. Horiz. 7(6), 1519-1527 (2020). https://doi.org/10.1039/d0mh00495b

29. W. Kosaka, Z. Liu, J. Zhang, Y. Sato, A. Hori et al., Gasresponsive porous magnet distinguishes the electron spin of molecular oxygen. Nat. Commun. 9, 5420 (2018). https://doi. org/10.1038/s41467-018-07889-1

30. J. Luo, T. Dziubla, R. Eitel, A low temperature co-fired ceramic based microfluidic Clark-type oxygen sensor for realtime oxygen sensing. Sens. Actuators B 240, 392-397 (2017). https://doi.org/10.1016/j.snb.2016.08.180

31. A.V. Agrawal, N. Kumar, M. Kumar, Strategy and future prospects to develop room-temperature-recoverable $\mathrm{NO}_{2}$ gas sensor based on two-dimensional molybdenum disulfide. Nano-Micro Lett. 13, 38 (2021). https://doi.org/10.1007/ s40820-020-00558-3

32. K. Kadimisetty, S. Malla, J.F. Rusling, Automated 3-D printed arrays to evaluate genotoxic chemistry: e-cigarettes and water samples. ACS Sens. 2(5), 670-678 (2017). https://doi.org/10. 1021/acssensors.7b00118

33. Z. Li, X. Liu, M. Zhou, S. Zhang, S. Cao et al., Plasmainduced oxygen vacancies enabled ultrathin $\mathrm{ZnO}$ films for highly sensitive detection of triethylamine. J. Hazard. Mater. 415, 125757 (2021). https://doi.org/10.1016/j.jhazmat.2021. 125757

34. A.V. Raghu, K.K. Karuppanan, B. Pullithadathil, Highly sensitive, temperature-independent oxygen gas sensor based on anatase $\mathrm{TiO}_{2}$ nanoparticle grafted, $2 \mathrm{D}$ mixed valent $\mathrm{VO}_{\mathrm{x}}$ nanoflakelets. ACS Sens. 3(9), 1811-1821 (2018). https://doi.org/ 10.1021/acssensors. 8b00544

35. M.A. Stoeckel, M. Gobbi, S. Bonacchi, F. Liscio, L. Ferlauto et al., Reversible, fast, and wide-range oxygen sensor based on nanostructured organometal halide perovskite. Adv. Mater. 29(38), 1702469 (2017). https://doi.org/10.1002/adma.20170 2469

36. H. Wang, L. Chen, J. Wang, Q. Sun, Y. Zhao, A micro oxygen sensor based on a nano sol-gel $\mathrm{TiO}_{2}$ thin film. Sensor 14(9), 16423-16433 (2014). https://doi.org/10.3390/s140916423

37. Y. Xiong, W. Lu, D. Ding, L. Zhu, X. Li et al., Enhanced room temperature oxygen sensing properties of $\mathrm{LaOCl}-\mathrm{SnO}_{2}$ hollow spheres by UV light illumination. ACS Sens. 2(5), 679-686 (2017). https://doi.org/10.1021/acssensors.7b00129

38. S. Xu, H. Fu, Y. Tian, T. Deng, J. Cai et al., Exploiting twodimensional $\mathrm{Bi}_{2} \mathrm{O}_{2} \mathrm{Se}$ for trace oxygen detection. Angew. Chem. Int. Ed. 59(41), 17938-17943 (2020). https://doi.org/ 10.1002/anie. 202006745

39. X. Liu, T. Ma, N. Pinna, J. Zhang, Two-dimensional nanostructured materials for gas sensing. Adv. Funct. Mater. 27(37), 1702168 (2017). https://doi.org/10.1002/adfm.20170 2168
40. Y. Shi, M. Wang, C. Hong, Z. Yang, J. Deng et al., Multijunction joints network self-assembled with converging $\mathrm{ZnO}$ nanowires as multi-barrier gas sensor. Sens. Actuators B 177, 1027-1034 (2013). https://doi.org/10.1016/j.snb.2012.11.084

41. J. Wu, Z. Wu, H. Xu, Q. Wu, C. Liu et al., An intrinsically stretchable humidity sensor based on anti-drying, self-healing and transparent organohydrogels. Mater. Horiz. 6(3), 595-603 (2019). https://doi.org/10.1039/c8mh01160e

42. X. Luo, M.Y. Akram, Y. Yuan, J. Nie, X. Zhu, Silicon dioxide/ poly(vinyl alcohol) composite hydrogels with high mechanical properties and low swellability. J. Appl. Polym. Sci. 136(1), 46895 (2019). https://doi.org/10.1002/app.46895

43. Y.Z. Zhang, K.H. Lee, D.H. Anjum, R. Sougrat, Q. Jiang et al., MXenes stretch hydrogel sensor performance to new limits. Sci. Adv. (2018). https://doi.org/10.1126/sciadv.aat0098

44. Z. Wu, W. Shi, H. Ding, B. Zhong, W. Huang et al., Ultrastable, stretchable, highly conductive and transparent hydrogels enabled by salt-percolation for high-performance temperature and strain sensing. J. Mater. Chem. C 9(39), 13668-13679 (2021). https://doi.org/10.1039/D1TC02506F

45. Y. Xu, L. Zhang, J. Xu, Y. Wei, X. Xu, Membrane permeability of the human pluripotent stem cells to $\mathrm{Me}_{2} \mathrm{SO}$, glycerol and 1,2-propanediol. Arch. Biochem. Biophys. 550-551, 67-76 (2014). https://doi.org/10.1016/j.abb.2014.04.010

46. D. Bao, Z. Wen, J. Shi, L. Xie, H. Jiang et al., An anti-freezing hydrogel based stretchable triboelectric nanogenerator for biomechanical energy harvesting at sub-zero temperature. J. Mater. Chem. A 8(27), 13787-13794 (2020). https://doi.org/ 10.1039/d0ta03215h

47. W. Ge, S. Cao, Y. Yang, O.J. Rojas, X. Wang, Nanocellulose/ $\mathrm{LiCl}$ systems enable conductive and stretchable electrolyte hydrogels with tolerance to dehydration and extreme cold conditions. Chem. Eng. J. 408, 127306 (2021). https://doi.org/10. 1016/j.cej.2020.127306

48. H. Liao, X. Guo, P. Wan, G. Yu, Conductive MXene nanocomposite organohydrogel for flexible, healable, low-temperature tolerant strain sensors. Adv. Funct. Mater. 29(39), 1904507 (2019). https://doi.org/10.1002/adfm.201904507

49. X.P. Morelle, W.R. Illeperuma, K. Tian, R. Bai, Z. Suo et al., Highly stretchable and tough hydrogels below water freezing temperature. Adv. Mater. 30(35), e1801541 (2018). https://doi. org/10.1002/adma.201801541

50. F. Chen, D. Zhou, J. Wang, T. Li, X. Zhou et al., Rational fabrication of anti-freezing, non-drying tough organohydrogels by one-pot solvent displacement. Angew. Chem. Int. Ed. 57(22), 6568-6571 (2018). https://doi.org/10.1002/anie.201803366

51. T. Wang, S. Gunasekaran, State of water in chitosan-PVA hydrogel. J. Appl. Polym. Sci. 101(5), 3227-3232 (2006). https://doi.org/10.1002/app.23526

52. Y. Xu, L. Zheng, C. Yang, X. Liu, J. Zhang, Highly sensitive and selective electronic sensor based on Co catalyzed $\mathrm{SnO}_{2}$ nanospheres for acetone detection. Sens. Actuators B 304, 127237 (2020). https://doi.org/10.1016/j.snb.2019.127237

53. A. Shrivastava, V. Gupta, Methods for the determination of limit of detection and limit of quantitation of the analytical 
methods. Chron. Young Sci. 2(1), 1 (2011). https://doi.org/ 10.4103/2229-5186.79345

54. J. Wu, Z. Wu, W. Huang, X. Yang, Y. Liang et al., Stretchable, stable, and room-temperature gas sensors based on selfhealing and transparent organohydrogels. ACS Appl. Mater. Interfaces 12(46), 52070-52081 (2020). https://doi.org/10. 1021/acsami.0c17669

55. X. Pan, Q. Wang, P. He, K. Liu, Y. Ni et al., A bionic tactile plastic hydrogel-based electronic skin constructed by a nervelike nanonetwork combining stretchable, compliant, and selfhealing properties. Chem. Eng. J. 379, 122271 (2020). https:// doi.org/10.1016/j.cej.2019.122271

56. X. Pei, H. Zhang, Y. Zhou, L. Zhou, J. Fu, Stretchable, selfhealing and tissue-adhesive zwitterionic hydrogels as strain sensors for wireless monitoring of organ motions. Mater. Horiz. 7(7), 1872-1882 (2020). https://doi.org/10.1039/d0mh0 $0361 \mathrm{a}$

57. C. Shao, M. Wang, L. Meng, H. Chang, B. Wang et al., Mussel-inspired cellulose nanocomposite tough hydrogels with synergistic self-healing, adhesive and strain sensitive properties. Chem. Mater. 30(9), 3110-3121 (2018). https://doi.org/ 10.1021/acs.chemmater.8b01172

58. J. Yang, R. Bai, B. Chen, Z. Suo, Hydrogel adhesion: a supramolecular synergy of chemistry, topology, and mechanics. Adv. Funct. Mater. 30(2), 1901693 (2019). https://doi.org/10. 1002/adfm.201901693

59. Q. Liu, G. Nian, C. Yang, S. Qu, Z. Suo, Bonding dissimilar polymer networks in various manufacturing processes. Nat. Commun. 9, 846 (2018). https://doi.org/10.1038/ s41467-018-03269-x

60. J. Yang, R. Bai, Z. Suo, Topological adhesion of wet materials. Adv. Mater. 30(25), 1800671 (2018). https://doi.org/10.1002/ adma.201800671

61. X. Li, J. Lai, Y. Deng, J. Song, G. Zhao et al., Supramolecular adhesion at extremely low temperatures: a combined experimental and theoretical investigation. J. Am. Chem. Soc. 142(51), 21522-21529 (2020). https://doi.org/10.1021/jacs. 0c10786
62. C. Yan, J. Wang, X. Wang, W. Kang, M. Cui et al., An intrinsically stretchable nanowire photodetector with a fully embedded structure. Adv. Mater. 26(6), 943-950 (2014). https://doi. org/10.1002/adma.201304226

63. R.S. Aga, D. Jowhar, A. Ueda, Z. Pan, W.E. Collins et al., Enhanced photoresponse in $\mathrm{ZnO}$ nanowires decorated with CdTe quantum dot. Appl. Phys. Lett. 91, 232108 (2007). https://doi.org/10.1063/1.2822896

64. Z. Wu, H. Ding, K. Tao, Y. Wei, X. Gui et al., Ultrasensitive, stretchable, and fast-response temperature sensors based on hydrogel films for wearable applications. ACS Appl. Mater. Interfaces 13(18), 21854-21864 (2021). https://doi.org/10. 1021/acsami.1c05291

65. G.G. Wildgoose, D. Giovanelli, N.S. Lawrence, R.G. Compton, High-temperature electrochemistry: a review. Electroanalysis 16(6), 421-433 (2004). https://doi.org/10.1002/elan. 200302875

66. S. Feng, Q. Li, S. Wang, B. Wang, Y. Hou et al., Tunable dual temperature-pressure sensing and parameter self-separating based on ionic hydrogel via multisynergistic network design. ACS Appl. Mater. Interfaces 11(23), 21049-21057 (2019). https://doi.org/10.1021/acsami.9b05214

67. N. Lopez-Ruiz, J. Lopez-Torres, M.A.C. Rodriguez, I.P. Vargas-Sansalvador, A. Martinez-Olmos, Wearable system for monitoring of oxygen concentration in breath based on optical sensor. IEEE Sens. J. 15(7), 4039-4045 (2015). https://doi. org/10.1109/jsen.2015.2410789

68. Z. Wu, W. Zhou, W. Deng, C. Xu, Y. Cai et al., Antibacterial and hemostatic thiol-modified chitosan-immobilized AgNPs composite sponges. ACS Appl. Mater. Interfaces 12(18), 20307-20320 (2020). https://doi.org/10.1021/acsami.0c05430

69. M.C. Darnell, J.Y. Sun, M. Mehta, C. Johnson, P.R. Arany et al., Performance and biocompatibility of extremely tough alginate/ polyacrylamide hydrogels. Biomaterials 34(33), 8042-8048 (2013). https://doi.org/10.1016/j.biomaterials.2013.06.061

70. F. Gottrup, Oxygen in wound healing and infection. World J. Surg. 28, 312-315 (2004). https://doi.org/10.1007/ s00268-003-7398-5 